\title{
The Nabidae (Insecta, Hemiptera, Heteroptera) of Argentina
}

\author{
Marcela Cornelis', María C. Coscarón ${ }^{2}$ \\ I Universidad Nacional de La Pampa. Facultad de Ciencias Exactas y Naturales. Uruguay 151 L6300CLB, \\ Santa Rosa, La Pampa. Argentina 2 Universidad Nacional de La Plata. Facultad de Ciencias Naturales y \\ Museo. División Entomología. Paseo del Bosque s/n 1900, La Plata, Buenos Aires. Argentina \\ Corresponding author: Marcela Cornelis (cornelismarcela@gmail.com), \\ María C. Coscarón (mcoscaron@fcnym.unlp.edu.ar)
}

Academic editor: Thomas Henry | Received 8 March 2013 | Accepted 14 August 2013 | Published 20 September 2013

Citation: Cornelis M, Coscarón MC (2013) The Nabidae (Insecta, Hemiptera, Heteroptera) of Argentina. ZooKeys 333: 1-30. doi: 10.3897/zookeys.333.5084

\begin{abstract}
In Argentina, five genera and 14 species are recorded in the subfamilies Prostemmatinae and Nabinae: Hoplistoscelis sordidus Reuter, Lasiomerus constrictus Champion, Metatropiphorus alvarengai Reuter, Nabis argentinus Meyer-Dür, Nabis (Tropiconabis) capsiformis Germar, Nabis faminei Stål, Nabis paranensis Harris, Nabis punctipennis Blanchard, Nabis roripes Stål, Nabis setricus Harris, Nabis tandilensis Berg, Pagasa (Pagasa) costalis Reuter, Pagasa (Lampropagasa) fuscipennis Reuter and Pagasa (Pagasa) signatipennis Reuter.
\end{abstract}

\section{Keywords}

Nabidae, key, Argentina, taxonomy, distribution

\section{Introduction}

The Nabidae, often called damsel bugs, are a small group of predatory insects of various shapes and colours, ranging from 5 to $15 \mathrm{~mm}$.

In the Neotropical catalogue, Volpi and Coscarón (2010) and Coscarón and Volpi (2013) provided a summary of the classification of the group and an exhaustive introduction to the literature. Two subfamilies, Nabinae and Prostemmatinae, comprising 11 genera and 83 species are recognized in the Neotropical Region and in Argentina four genera and 13 species (Coscarón, submitted). Although all of them are predators, some have been observed feeding on plants (Ridgway and Jones 1968, Stoner 1972, 
Kiman and Yergan 1985). The members of Nabinae feed on many different small arthropods including aphids, leafhoppers, larvae and adults of Hemiptera, thrips, flies, caterpillars, beetle larvae, eggs of insects, small spiders and acari, whereas those of Prostemmatinae appear to prey exclusively on other Heteroptera, especially Lygaeoidea (Pericart 1987, Lattin 1989). The predaceous habit, together with the widespread occurrence of some species in agroecosystems, has attracted the attention of entomologists (Lattin 1989). Although all known species are terrestrial, some have been found in moist areas on the ground or at the edge of streams, ponds, and marshes (both fresh and saline) (Lattin 1966, 1989, Pericart 1987).

Argentina, the geographical area considered in this paper, lies in the Neotropical Region. The country covers an area of $2,791,810 \mathrm{~km}^{2}$ and is bordered by Uruguay, Brazil, Paraguay, Bolivia, and Chile. Approximately $75 \%$ of the country is occupied by arid and semiarid areas, but rainforests are also present in the northeastern part of the country, for example the Yungas and Paranaense regions.

Knowledge of the South American fauna is poor, especially in relation to the taxa of economic importance, and no comprehensive keys for identification of the species living in the region have previously been published.

The objective of this paper is to provide an illustrated key of the genera of Nabidae from Argentina, including a diagnosis, geographical distribution, list of species for each genus, and a redescription when necessary.

\section{Materials and methods}

The specimens examined belong to the collections of the Museo de Ciencias Naturales de La Plata (MLP), La Plata, Buenos Aires, Argentina (http://www.fcnym.unlp.edu. ar/abamuse.html), the American Museum of Natural History (AMNH), New York, USA (http://www.amnh.org/) and the Swedish Museum of Natural History (NHRS), Stockholm, Sweden (http://www.nrm.se/).

Observations were made with a Leica MZ95 stereoscope and measurements with a micrometer eyepiece and expressed in millimeters $(\mathrm{mm})$. The male genitalia were dissected by removing the pygophore from the abdomen with a pair of forceps, and the female genitalia by cutting the genital plates with $12 \mathrm{~cm}$ dental standard straight scissors. Genitalia from both sexes were cleared in a $\mathrm{KOH}$ solution for 24 hours. All dissected structures were stained with methylene blue and photographed in glycerin. Images of adults and genitalia were taken with a digital camera (Kodak 3.1 megapixels) and a magnifying Wild M-Stereomicroscope. The terminology of the male and female genitalia follows Dupuis (1955) and Pericart (1987). The photographs were compared with material from the Naturhistoriska Riksmuseet of Stockholm, Sweden (http://www.nrm.se/2.1286b10fdbe80efba80001.html) and the American Museum of Natural History of New York (http://www.amnh. org/). Descriptions were taken from http://www.biodiversitylibrary.org/, http:// archive.org/. The diagnosis of the genus Pagasa, Metatropiphorus and Nabis were 
taken from Blatchley (1926) (http://www.biodiversitylibrary.org/item/29937); and the diagnosis of Lasiomerus and Hoplistoceslis from Champion (1899) (http:// www.biodiversitylibrary.org/item/14631\#page/311/mode/1up), Harris (1928) and Reuter (1890) (http://www.archive.org/search.php?query=Revue\%20 $\mathrm{d} \% \mathrm{C} 2 \% \mathrm{~B} 4$ Entomologie\%201890). Specimen locations were georeferenced using Google Earth 6.1.0.4738 (beta) (Google Inc. 2011). Location decimal degree coordinates were processed with DIVA-GIS 7.1.7 (http:// www.diva-gis.org/) to generate species distribution maps.

\section{Results}

\section{Key to species of Nabidae from Argentina}

1 Scutellum with 1-7 pairs of conspicuous trichobothria along lateral margins (Fig. 1c). Legs short and thick, fore femur usually strongly incrassate; labium relatively short and stout; antennae four segmented, plus one extra at the base of antennal segment II, which is half or more than half of the length of antennal segment I (Fig. 1b). Body shiny black. (Subfamily Prostemmatinae Reuter) ... 2

- Scutellum without trichobothria; legs long and thin, fore femur at most only moderately incrassate; rostrum relatively slender and elongate; antennae long with four segments, and sometimes an additional ring-shaped segment at the base of the second; not shiny black. (Subfamily Nabinae Costa) .................. 4

2 Rostral segment II shorter than III, not extending beyond hind margins of eyes (Subgenus Lampropagasa Reuter) ........ Pagasa fuscipennis Reuter (Fig. 1a-k)

- $\quad$ Rostral segment II longer than III, extending distinctly beyond hind margins of eyes (Subgenus Pagasa Stål) .3

3 Rostrum reaching from the middle to the apex of fore coxae. Fore trochanter with 4-6 minute black teeth, middle and hind trochanters without teeth. Fore femur strongly thickened Pagasa costalis Reuter

- $\quad$ Rostrum reaching the middle of the mesothorax. Fore, middle and hind trochanters without teeth. Fore femur moderately thickened.

Pagasa signatipennis Reuter

First antennal segment twice as long as the head and thickened on apical third Metatropiphorus alvarengai Reuter

- $\quad$ First antennal segment never twice the long of the head and not thickened apically .5

Fore and middle legs with rows of long and rigid spines (Fig. 2a)

Lasiomerus constrictus Champion (Fig. 3)

Fore and middle legs without rows of long and rigid spines

6 Fore and middle femora armed beneath with minute, short, rather blunt piceous teeth (Fig. 2b) Hoplistoscelis sordida Reuter (Fig. 4a-c)

- $\quad$ Fore and middle femora with short dense setae shiny, without teeth (Fig. 2c) ...7 
7 Body robust and shining, sparsely covered with fine, whitish pubescence...... Nabis tandilensis Berg (Fig. 5)

- $\quad$ Body not robust and shining, covered with abundant whitish pubescence...8 8

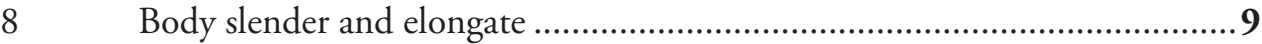

- Body not slender and elongate ................................................. 10

9 Hemelytra clear and hyaline. Second segment of rostrum slightly longer than the third. Pronotum slightly longer than broad (Subgenus Tropiconabis Kerzhner) Nabis capsiformis Germar (Fig. 6a-j)

- Hemelytra not clear and hyaline. Second segment of rostrum slightly shorter than the third. Pronotum slightly broader than long... Nabis setricus Harris

10 Venter uniformly sordid brown. Hemelytra short in brachypterous form, reaching onto the middle of the first dorsal segment of the abdomen.....Nabis roripes Stål

- Venter not sordid brown. Hemelytra in brachypterous form surpassing the middle of the first dorsal segment of the abdomen ................................11

11 Length of first antennal segment equal to or slightly shorter than the distance between the eyes. Length of second antennal segment subequal to the width of the base of the pronotum. Membrane in brachypterous form slightly surpassing the apex of the corium Nabis paranensis Harris (Fig. 7a-i)

- $\quad$ Length of first antennal segment markedly shorter than the distance between the eyes. Second antennal segment shorter than the width of the base of the pronotum. Membrane in brachypterous form widely surpassing the apex of the corium ...................................................................... 12

12 Pronotum in macropterous form greatly expanded behind, a third wider than long. Pronotum in brachypterous form visibly wider than long, but strongly compressed on the sides between the anterior and posterior lobes

Nabis argentinus Meyer-Dür (Fig. 8a-i)

- $\quad$ Pronotum in macropterous form not more than one fifth wider than long. Pronotum in brachypterous form as wide as long, anterior and posterior lobes not so markedly different...

13 Body and legs robust. Eyes large. Length of antennal segment II hardly equal to the width of the head across eyes ...............Nabis faminei Stål (Fig. 9a-i)

- $\quad$ Body and legs more slender. Eyes smaller. Length of antennal segment II slightly longer than width of the head across eyes....

Nabis punctipennis Blanchard (Fig. 10a-j)

\section{Subfamily Prostemmatinae Reuter, 1890 \\ Tribe Prostemmatini Reuter, 1890}

Genus Pagasa Stål, 1862

Diagnosis. Black or fuscous shining species, having the eyes large, prominent and coarsely granulated; pronotum longer than broad with a fine, straight, transverse 
groove very close to front margin; scutellum with two small median fovea; embolium of hemelytra present; front and middle tibiae with a spongy fossa at apex.

\section{Subgenus Lampropagasa Reuter, 1909}

Diagnosis. Rostral segment II shorter than or as long as segment III, in most species not surpassing hind margin of eye. Corium and clavus uniformly (strongly or moderately) shining throughout. Both veins of corium $(\mathrm{R}+\mathrm{M}$ and $\mathrm{Cu})$ or at least the inner vein $(\mathrm{Cu})$ distinct up to hind margin of corium. Vein $\mathrm{Cu}$ with punctures (obsolete in some species) on both sides.

\section{Pagasa fuscipennis Reuter, 1909}

http://species-id.net/wiki/Pagasa_fuscipennis

Figs $1 \mathrm{a}-\mathrm{k}, 13 \mathrm{a}$

Pagasa nitida Berg 1884. Annales de la Sociedad Científica Argentina 2: 105.

Pagasa fuscipennis Reuter and Poppius 1909. Acta Societatis Scientiarum Fennicae 37:

30. Pennington 1921. Lista de los Hemipteros de la Républica Argentina. Segunda Parte: 26. Harris 1939. Notas del Museo de La Plata 26: 369. Ruffinelli and Pirán 1959. Boletín de la Facultad de Agronomía de Montevideo 51: 41. Kerzhner and Konstantinov 2008. Zoosystematica Rossica 17: 38. Volpi and Coscarón 2010. Zootaxa 2513: 62.

Material examined. ARGENTINA: BUENOS AIRES: Prov. de Buenos Aires, J. Bosq. 1 q (MLP); Ciudad de Buenos Aires 34³6'30.30"S; 58²2'23.38"W, 25XII-1918 Bosq. col., 1 ๆ (MLP); Ciudad de Buenos Aires, 9-III-1914, 1 q (MLP);

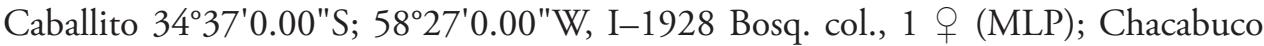
34³8'9.36"S; 60²7'54.44"W, F. Lynch col., Harris det. 1 q (MLP). CHACO: Km

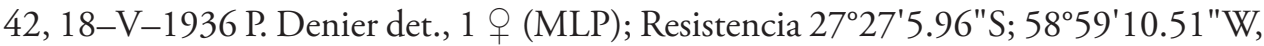
21-I-1939 Harris det. 1 † (MLP). SANTIAGO DEL ESTERO: Río Salado, Wagner col., 1 \& (MLP). URUGUAY: Montevideo, Colón 3448'8.30"S, 56²13'26.90"W, 12-I-1929 A. Montora col., Harris det., 1 q (MLP).

Distribution in Argentina. Buenos Aires: Caballito, Ciudad de Buenos Aires, Chacabuco, Ciudad de Bs. As., San Fernando, Tandil; Chaco: Resistencia; Chubut: Rio Turbio, El Maiten, Pedregos Epuyen; Córdoba: Alta Gracia Misiones; Río Negro: Norquinco, El Bolsón; Salta: Metan; San Luis; Santa Fé: Ciudad de Santa Fé.

Distribution outside Argentina. Brazil: Goyas, São Paulo, Minas Gerais, Santa Catarina; Paraguay: Asunción, Horqueta; Uruguay: Colonia, Montevideo, Soriano.

Measurements. Female $(\mathrm{n}=5)$ : Length 5.00-6.36 (mean = 5.96). Head: length $0.82-0.90($ mean $=0.85)$, width 0.82-0.91 (mean = 0.89); eye width $0.41-0.48$ (mean $=0.45)$, interocular width $0.29-0.33$ (mean $=0.30)$. Rostrum: ratio of segment lengths 


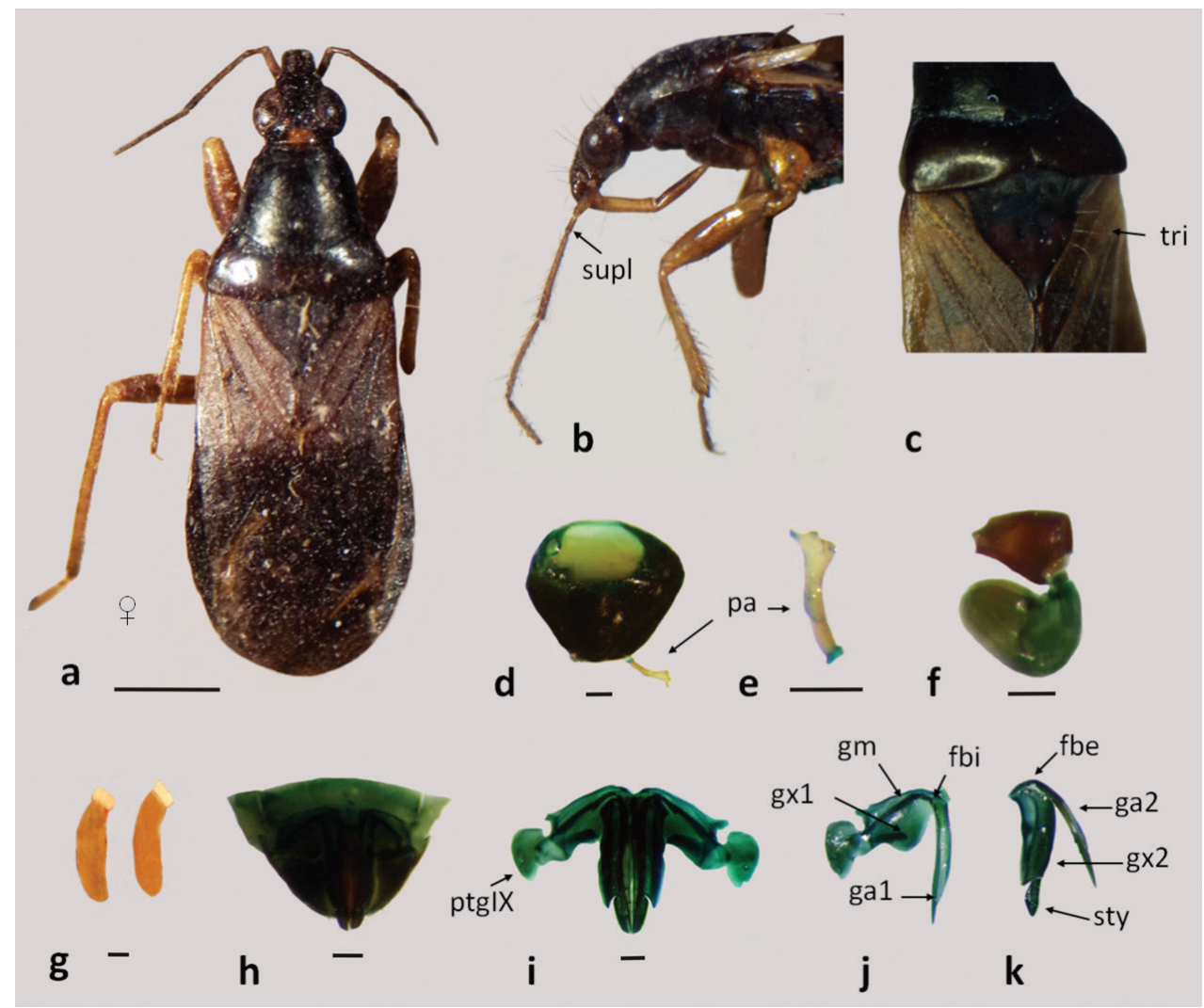

Figure I. Pagasa fuscipennis: a dorsal view $\mathbf{b}$ lateral view $\mathbf{c}$ scutellum with trichobotrias; $\mathbf{d}-\mathbf{f}$ male genitalia: $\mathbf{d}$ pygophore $\mathbf{e}$ paramere $\mathbf{f}$ aedeagus $\mathbf{g}$ eggs $\mathbf{h}-\mathbf{k}$ female genitalia: $\mathbf{h}-\mathbf{i}$ genital segment $\mathbf{j}$ first gonapophysis and gonocoxite $1 \mathbf{k}$ second gonapophysis and gonocoxite 2. (fbe: external fibula; fbi: internal fibula, ga1 and ga2: gonapophysis 1 and 2; gm: gonangulum; gxp1 and 2: gonocoxites 1 and 2, pa: paramere, ptgIX: paratergite IX; sty: styloid; supl: supplementary; tri: trichobotria.). Figures a-c scale line $1 \mathrm{~mm}$; $\mathbf{d}-\mathbf{k}$ scale line $0.2 \mathrm{~mm}$.

about $1: 1.96: 2.28: 1$. Antenna: ratio of segment about $1: 0.57: 2.48: 2.27: 2.21$. Pronotum length $1.35-1.59($ mean $=1.47)$, width $1.69-1.88($ mean $=1.77)$. Hemelytra length 2.07-4.05 (mean = 3.47). Abdomen: length 2.17-2.66 (mean = 2.47), width 1.93-2.18 (mean = 2.07). Legs: fore femora: length $1.24-1.35($ mean $=1.31)$, width $0.42-0.53$ (mean $=0.47)$; middle femora: length $1.21-1.40($ mean $=1.33)$, width $0.27-0.33$ (mean $=0.30)$; hind femora $1.78-1.83($ mean $=1.81)$, width 0.29 $0.33($ mean $=0.31)$. Fore tibiae: length 1.03-1.25 (mean = 1.12), width $0.21-0.29$ $($ mean $=0.27)$; middle tibiae: length $1.11-1.35$ (mean = 1.24), width 0.14-0.19 (mean $=0.15)$; hind tibiae: length 1.87-2.12 (mean = 2.01), width 0.09-0.17 (mean = 0.13).

Eggs (Fig. 1g). Length 1.08-1.12 (mean = 1.10; $\mathrm{n}=4$ ), width $0.28-0.36$ (mean = 0.31; $\mathrm{n}=4$ ). Microsculpture absent. Mature eggs were taken from dissected female oviducts.

New record. Argentina: La Pampa, Santa Rosa: 36³7'17.41"S; 64²17'5.91"W, Cornelis col. 


\section{Subgenus Pagasa Stål, 1862}

Diagnosis. Rostral segment II longer than or as long as segment III and surpassing hind margin of eye. At least inner half of clavus and inner corner of corium dull, differing from the shining outer part of hemelytron; in most species, the shining part occupies only the outer part of corium outside the medial fracture. Only vein $\mathrm{Cu}$ on corium distinct, but obsolete or lacking in hind part of corium. Usually, vein $\mathrm{Cu}$ with punctures on outer side only.

\section{Pagasa costalis Reuter, 1909}

Pagasa costalis Reuter in Reuter and Poppius 1909. Acta Societatis Scientiarum Fennicae 37: 26-29. Harris 1939. Notas del Museo de La Plata 26: 368. Ruffinelli and Pirán 1959. Boletín de la Facultad de Agronomía de Montevideo, 51: 40. Kerzhner and Konstantinov 2008. Zoosystematica Rossica, 17: 48. Volpi and Coscarón 2010. Zootaxa, 2513: 63.

Distribution in Argentina. Buenos Aires, Salta.

Distribution outside Argentina. Ecuador: Milagro; Paraguay: Asunción; Surinam: Saramacca; Uruguay: Montevideo.

\section{Pagasa signatipennis Reuter, 1909}

http://species-id.net/wiki/Pagasa_signatipennis

Pagasa signatipennis Reuter and Poppius 1909. Acta Societatis Scientiarum Fennicae 37:

26. Harris 1939. Notas del Museo de La Plata 26: 369. Kerzhner and Konstantinov 2008. Zoosystematica Rossica 17: 48. Volpi and Coscarón 2010. Zootaxa 2513: 64.

Distribution in Argentina. Formosa.

Distribution outside Argentina. Bolivia: Rosario, Villa Vicencio; Brazil: Mato Grosso, Santarem; Colombia; Paraguay: Gran Chaco, Horqueta; Surinam: Kwatta, Wagenijen; Venezuela: Sarán de Aquae Apuae.

\section{Subfamily Nabinae Costa, 1853 \\ Tribe Nabini Costa, 1853}

Genus Metatropiphorus Reuter, 1872

http://species-id.net/wiki/Metatropiphorus

Diagnosis. Elongate, narrow finely pubescent species having the head behind eyes constricted to form a long cylindrical neck; vertex with two fine median grooves; rostrum reaching front coxae; pronotum about as wide at base as long, strongly constrict- 
ed behind middle, front lobe with a narrow median carina, and side margins distinct; hemelytra surpassing tip of abdomen, membrane large without closed discal cells; fore femora feebly swollen, armed beneath with a number of short distinct spines; fore tibiae setose beneath, apices obliquely truncate.

\section{Metatropiphorus alvarengai Kerzhner, 1987}

http://species-id.net/wiki/Metatropiphorus_alvarengai

Figs $13 \mathrm{~b}$

Metatropiphorus alvarengai Kerzhner 1987. Journal New York Entomological Society 95: 569. Holotype (AMNH). Volpi and Coscarón 2010. Zootaxa 2513: 57.

Holotype. http://research.amnh.org/iz/types_db/details.php?specimen_id=5458

Distribution in Argentina. Buenos Aires: Tigre, San Fernando.

Distribution outside Argentina. Brazil: Bahia, Mato Grosso, Santa Catarina. Surinam: Moengo.

\section{Genus Lasiomerus Reuter, 1890}

Diagnosis. Posterior lobe of pronotum strongly punctate; hemelytra distinctly constricted before the middle, the costal margin ciliate; femora annulate before the apex; posterior tibiae clothed with long, suberect setae.

\section{Lasiomerus constrictus (Champion, 1899)}

http://species-id.net/wiki/Lasiomerus_constrictus

Figs 2a, 3, 13c

Nabis (Hoplistoscelis) constrictus Champion 1899. Biologia Centrali-Americana 2: 303. Nabis constrictus Blatchley 1926. Heteroptera or True Bugs of Eastern North America,

with especial reference to the faunas of Indiana and Florida: 596.

Nabis (Lasiomerus) constrictus Harris 1928. Entomologica Americana 9: 51.

Lasiomerus constrictus Henry and Lattin 1988. Catalog of the Heteroptera, or True

Bugs, of Canada and the Continental United States, p 512. Volpi and Coscarón 2010. Zootaxa 2513: 57.

Material examined. ARGENTINA: BUENOS AIRES: San Isidro 34²8'14.98"S 58³1'43.00"W, 1 ㅇ (NHRS).

Distribution in Argentina. Buenos Aires: San Isidro.

Distribution outside Argentina. Guatemala; Honduras; México: Atoyac, Teapa; Panama: Volcan de Chiriqui. México to Panama. 


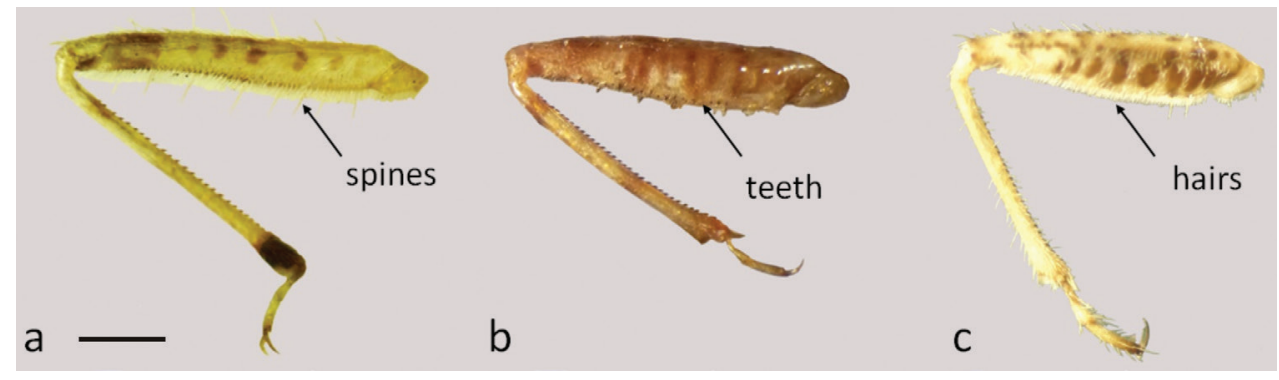

Figure 2. Fore leg: a Lasiomerus constrictus b Hoplistoscelis sordida c Nabis argentinus. Scale line $0.05 \mathrm{~mm}$.

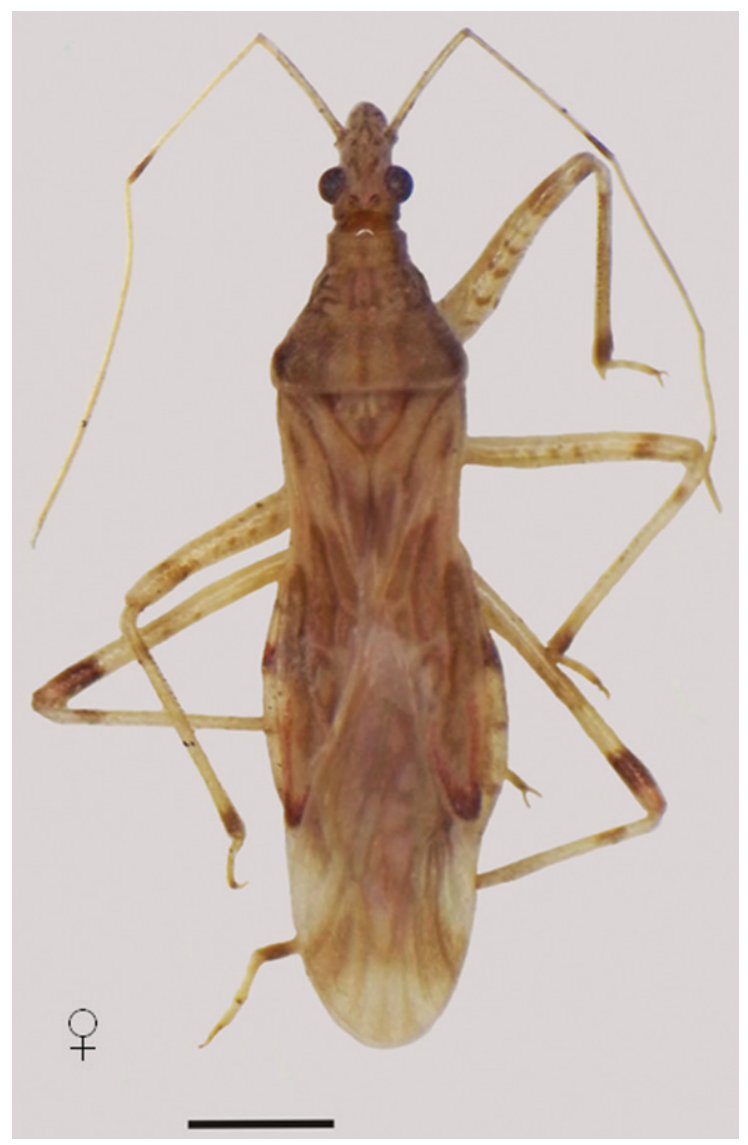

Figure 3. Lasiomerus constrictus dorsal view. Scale line $1 \mathrm{~mm}$.

Measurements. Female $(n=1)$ : Length 6.88. Head: length 0.83 , width 0.67 ; eye width 0.31 , interocular width 0.26 . Rostrum: ratio of segment lengths about $1: 3.86$ : $3.47: 2.08$. Antenna: ratio of segment about $1: 1.56: 1.56: 1.45$. Pronotum length 1.19 , width 1.40 . Hemelytra length 4.68. Abdomen: length 3.12 , width 1.76. Legs: fore femora: length 1.82 , width 0.26 ; middle femora: length 1.71 , width 0.20 ; hind 
femora 2.34 , width 0.15 . Fore tibiae: length 1.66 , width 0.10 ; middle tibiae: length 1.71, width 0.10; hind tibiae: length 2.96, width 0.078 .

Description. Body elongated and light brown. Head covered with abundant long white setae; area between eyes and antennae, and lateral anteocular region brown. Rostrum reaching middle coxae. Antennae long with setae, segment II with a distal band dark brown band; segments III and IV darker than I and II.

New records. 1 q: Argentina: Buenos Aires: San Isidro 34²8'14.98"S, $58^{\circ} 31^{\prime} 43.00^{\prime \prime}$, (NHRS).

\section{Genus Hoplistoscelis Reuter, 1890}

Diagnosis. Anterior and intermediate femora armed beneath with minute short, rather blunt, piceous teeth; tibiae annulate through out the entire length.

\section{Hoplistoscelis sordida (Reuter, 1872)}

http://species-id.net/wiki/Hoplistoscelis_sordida

Figs $2 \mathrm{~b}, 4 \mathrm{a}-\mathrm{c}$

Nabis sordidus Reuter 1872. Öfversigt af Kongliga Svenska Vetenskaps-Akademiens Förhandlingar 29: 85. Holotype (NHRS). Champion 1899. Biologia CentraliAmericana 2: 303. Harris 1930. Annals of the Carnegie Museum 19: 241-248. Hoplistoscelis sordida Kerzhner 1993. Zoosystematica Rossica 1:39. Volpi and Coscarón 2010. Zootaxa 2513: 56.

Holotype: http://www2.nrm.se/en/het_nrm/s/hoplistoscelis_sordidus.html

Material examined. USA: Iowa: Ames 42¹'38"N, 9337'54"W, 12-X-1926 H. M. Harris col., Harris det. 1 + (MLP); Davenport 4133'15"N, 90³6'14"W, 31-VIII1927 H. G Johnston col., Harris det. 2 ô ô (MLP).

Distribution outside Argentina. Brazil. Central and South America from the central part of México to Argentina. Costa Rica: Volcán de Iraza. Eastern North America. Guatemala: Vera Paz, Capetillo. México: Atoyac, Cuernavaca, Guerrero, Orizaba, San Marcos, Teapa, Vera Cruz. Panama: Volcán de Chiriqui. West Indies.

Observations. This species is not mapped because the exact place is not specified, according to Kerzhner (1993) "it occurs in Central and South America from central part of Mexico to Argentina".

Measurements. Male $(n=2)$ : Length 6.24-6.60 (mean = 6.42). Head: length $0.90-1.01$ (mean $=0.95$ ), width 0.82 ; eye width 0.37 , interocular width $0.30-0.33$ (mean $=0.31$ ). Rostrum: ratio of segment lengths about $1: 2.45: 2.63: 1.36$. Antenna: ratio of segment about 1: 1.60: 1.38: 1.34. Pronotum length 1.30 , width 1.45-1.60 $($ mean $=1.52)$. Hemelytra length 3.91-4.44 (mean = 4.17). Abdomen: length 3.00$3.18($ mean $=3.09)$, width $1.65-1.93($ mean $=1.79)$. Legs: fore femora: length $1.84-$ 


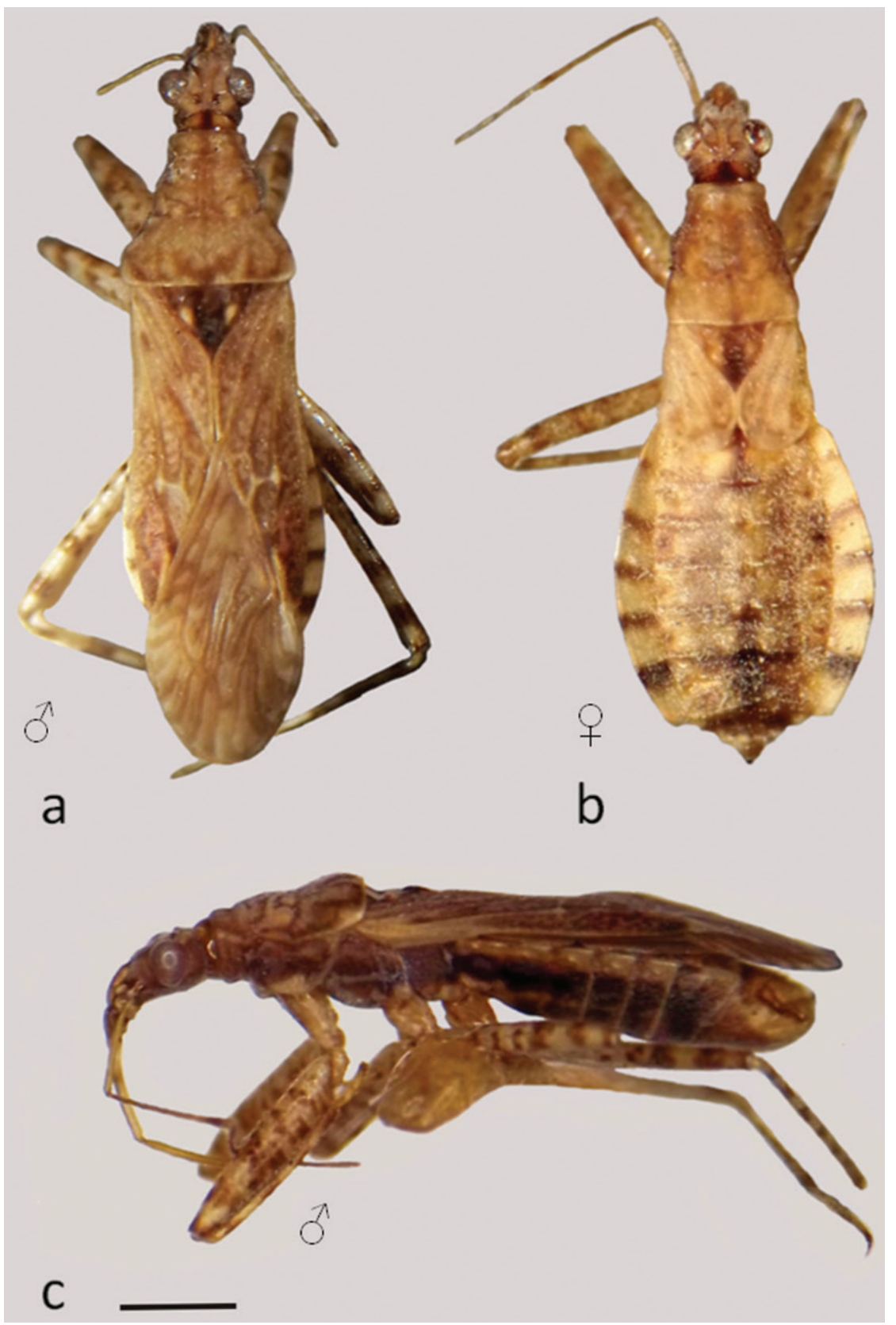

Figure 4. Hoplistoscelis sordida $\mathbf{a}-\mathbf{b}$ dorsal view c lateral view. Scale line $1 \mathrm{~mm}$.

$1.88($ mean $=1.86)$, width 0.37); middle femora: length 1.70-1.76 (mean = 1.73), width 0.26; hind femora 2.00-2.25 (mean = 2.12), width $0.18-0.20$ (mean $=0.19)$. Fore tibiae: length 1.54, width 0.11 ; middle tibiae: length $1.61-1.70$ (mean $=1.65$ ), width 0.075 ; hind tibiae: length 2.44-2.60 (mean = 2.52), width 0.075. 
Female $(\mathrm{n}=1)$ : Length 6.06 . Head: length 0.82 , width 0.86 ; eye width 0.41 , interocular width 0.35 . Rostrum: ratio of segment lengths about $1: 2: 3.03: 4.39$. Antenna: ratio of segment about $1: 1.40: 1.22: 1.23$. Pronotum length 1.27 , width 1.20 . Hemelytra length 1.12. Abdomen: length 2.70, width 2.25. Legs: fore femora: length 1.88, width 0.41 ; middle femora: length 1.76 , width 0.26 ; hind femora 2.30 , width 0.22 . Fore tibiae: length 1.57 , width 0.13 ; middle tibiae: length 1.70 , width 0.11 ; hind tibiae missing.

Genus Nabis Latreille, 1802

http://species-id.net/wiki/Nabis

Diagnosis. Body elongate or oblong-oval, usually slender. Pronotum campanulate with two fine transverse constrictions, humeral angles rounded, hind margin subtruncate; scutellum small, triangular, apex obtuse; clavus widened behind; hemelytra entire reaching or surpassing tip of abdomen, or abbreviated reaching only second dorsal segment, membrane with three elongate cells, their terminal bounding vein with numerous short veins radiating to tip of membrane; connexivum in males of macropterous forms usually narrowly or not at all exposed, in females more broadly so; front femora spindleshaped, moderately swollen, femora and tibiae beset beneath with numerous fine setae; front and middle tibiae with a short spongy lobe at apex; tarsi 3-jointed.

\section{Nabis tandilensis (Berg, 1884)}

http://species-id.net/wiki/Nabis_tandilensis

Figs $5,13 \mathrm{~d}$

Coriscus tandilensis Berg 1884. Annales de la Sociedad Científica Argentina 2: 106.

Nabis tandilensis Pennington 1921. Lista de los Hemipteros de la República Argentina.

Segunda Parte: 26. Harris 1939. Notas del Museo de La Plata 26: 377. Holotype

(MLP). Volpi and Coscarón 2010. Zootaxa 2513: 59.

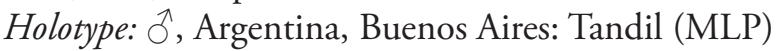

Material examined. Holotype $\sigma^{\lambda}$ BUENOS AIRES: Tandil $37^{\circ} 19^{\prime} 4.12 " \mathrm{~S} ; 59^{\circ}$ 9'1.41"W, 5-XI-1983 Doctor Holmberg, (MLP).

Distribution in Argentina. Buenos Aires: Tandil.

\section{Subgenus Tropiconabis Kerzhner, 1968}

Diagnosis. Body long and narrow, antennae and legs long, hemelytra surpassing tip of abdomen. Abdomen usually with clear edges. Paramere small. 


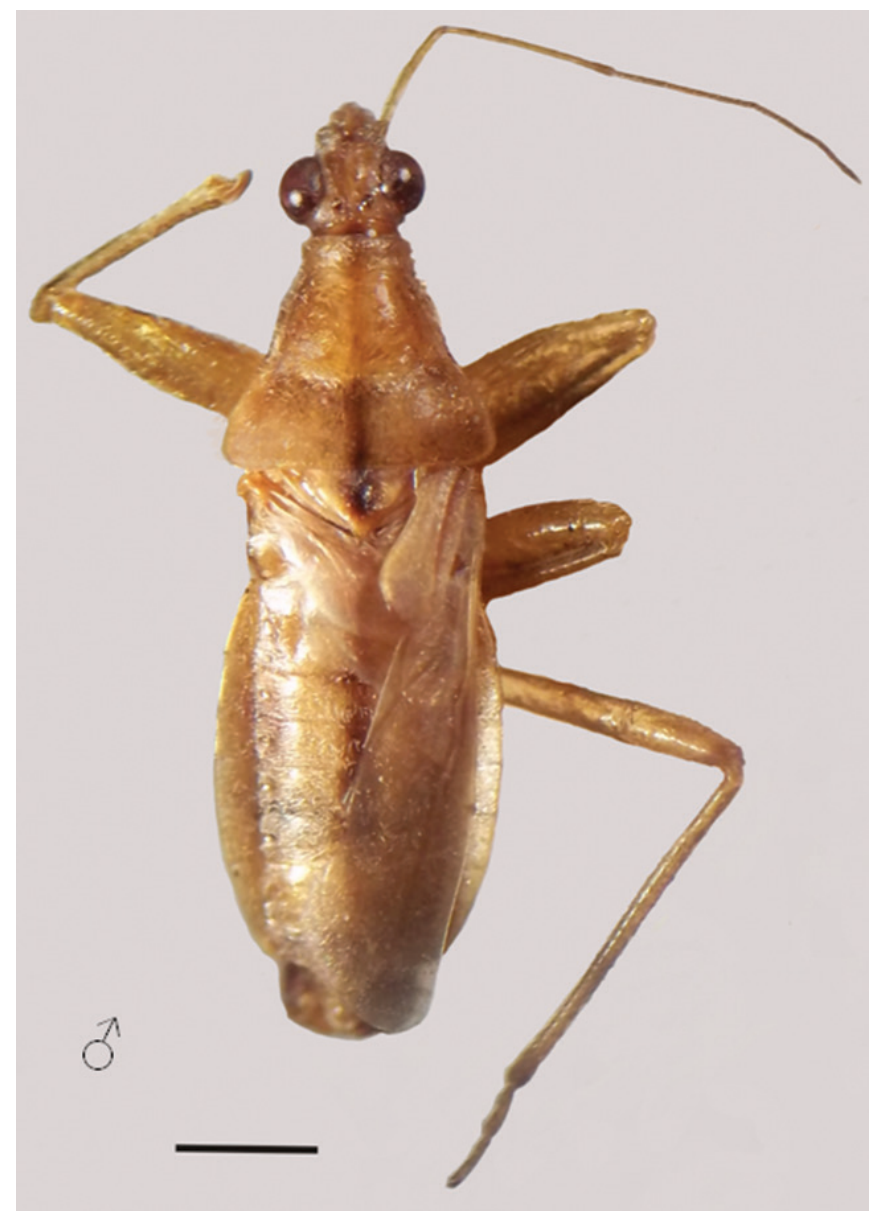

Figure 5. Nabis tandilensis dorsal view. Scale line $1 \mathrm{~mm}$.

\section{Nabis capsiformis Germar, 1837}

http://species-id.net/wiki/Nabis_capsiformis

Figs 6a-j, 13d

Nabis capsiformis Germar 1837. Silbermann's Revue Entomologique 5: 132. Pennington 1920-1921. Lista de los Hemípteros Heterópteros de la República Argentina. Segunda Parte: 26. Harris 1930. Annals of the Carnegie Museum Argentina 19: 246. Harris 1939. Notas del Museo de La Plata 26: 376. Ruffinelli and Pirán 1959. Boletín de la Facultad de Agronomía de Montevideo 51: 40. Prado 2008. Boletín del Museo Nacional de Historia Natural Chile 57: 44. Volpi and Coscarón 2010. Zootaxa 2513: 60.

Nabis elongatus Meyer-Dür 1870. Mitteilungen der Schweizerischen Entomologischen Gesellschaft 3:178. Synonymized by Reuter 1908: 114. 
Nabis kinbergii Reuter 1872. Öfversigt af Kongliga Svenska Vetenskaps-Akademiens Forhandlingar 29: 90. Synonymized by Reuter 1908: 114.

Coriscus capsiformis Stål 1873. Kongliga Svenska Vetenskaps-Akademiens Handlingar 11: 113 .

Coriscus elongatus Stål 1873. Kongliga Svenska Vetenskaps-Akademiens Handlingar 11: 114.

Coriscus kinbergii Stål 1873. Kongliga Svenska Vetenskaps-Akademiens Handlingar 11: 113 .

Reduviolus capsiformis Reuter 1908 Mémoires de la Société Entomologique de Belgique 15: 114.

Material examined. BUENOS AIRES: Bs. As. 1 q (MLP); J. Bosq col., 1 q (MLP); 1776 Harris det., 1 o (MLP); Alm. Brown 3450'24.22"S; 58²3'40.24"W, 19-V-36 1 ㅇ (MLP); Arrecifes 34 3'49.96"S; 60 6'12.56"W, 17-I-1939 Biraben-Scott leg. col., 1 q (MLP); Ciudad de Buenos Aires 34³6'30.30"S; 58²2'23.38"W, XI-1918 1 ㅇ (MLP), 12-II-1912 1 ㅇ (MLP); José C. Paz 34³0'54.38"S; 5845'58.49"W, XI-1958 1 क (MLP), XII-1911 1 ð (MLP), 1940 J. A. Rosas Costa leg. col., 1 ○,

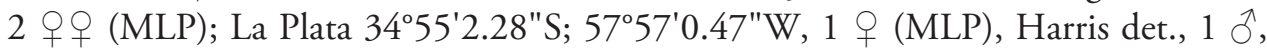

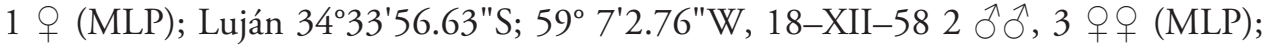
Mar del Plata 3758'47.49"S; 57³5'23.26"W, 5-XII-1938 Biraben-Scott leg. col.,

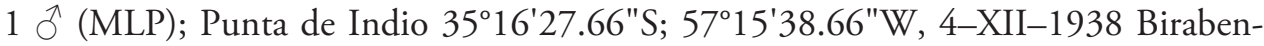
Scott leg. col., 2 우 (MLP); Rincón de Noario 8-IX-1935, 1 q (MLP); San Nicolas

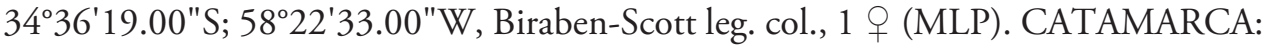
Pomán 28²3'47.29"S; 66'13'7.56"W, 8-III-62 Torres-Ferreyra col., 1 q (MLP). CORDOBA: Alta Gracia 31³9' 16.39"S; 64²5'50.17"W, I-35 C. Bruch leg. col., 1 o (MLP); Cabana $31^{\circ} 13^{\prime} 0.01$ "S; 64²2' $0.01^{\prime \prime} \mathrm{W}, 03-\mathrm{I}-1926$ Harris det., 1 क (MLP), 10-XI-1942 Biraben col., 1 ô (MLP), Marull 3059'45.16"S; 6249'37.61"W, 22-I-1940 Biraben, 1 o (MLP); San Antonio de Arredondo 31 28'57.22"S; 64³1'25.50"W, 14-II-1940 Biraben col., 1 ○, 1 ㅇ (MLP). CORRIENTES: Harris det., 1 specimen (without abdomen) (MLP); I-1921 De Carlo col., 1 ô (MLP);

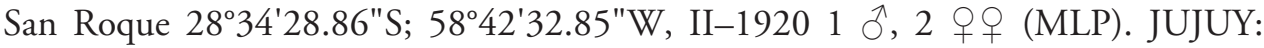
Pampa Blanca 24³1'58.57"S; 65 4'24.57"W, 13-III-1939 Biraben-Scott leg. col.,

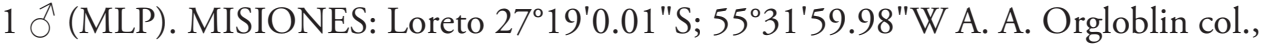
2 웅 (MLP). SANTIAGO DEL ESTERO: Girardet $27^{\circ} 37^{\prime} 0.02^{\prime \prime} \mathrm{S} ; 62^{\circ} 10^{\prime} 0.02^{\prime \prime} \mathrm{W}$, 9-XII-1939 Biraben-Bezzi, 2 우우 (MLP); Quimilí 27³8'39.06"S; 62²4'56.03"W, 9-XII-1939 Biraben-Bezzi col., 1 \& (MLP).

Distribution in Argentina. Buenos Aires: Alm. Brown, Arrecifes, Ciudad de Buenos Aires, José C. Paz, La Plata, Luján, Mar del Plata, Punta de Indio, Rincón de Noario, San Nicolas; Catamarca: Pomán; Córdoba: Alta Gracia, Cabana, Marull, San Antonio de Arredondo; Corrientes: San Roque; Jujuy: Pampa Blanca; La Pampa: Winifreda; Misiones: Loreto, Río Bermejo, Salto; Salta (Río Bermejo); Santiago del Estero: Girardet, Quimilí. 


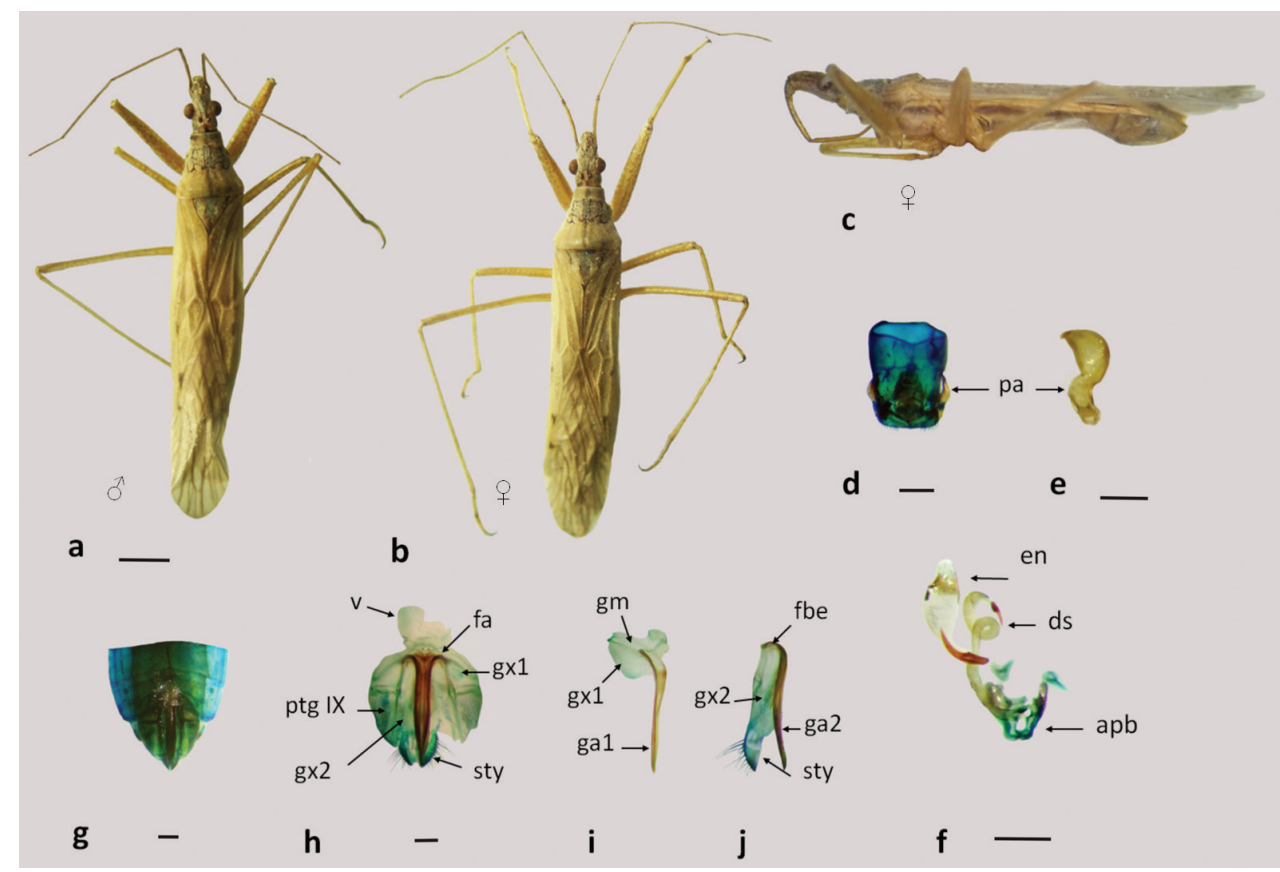

Figure 6. Nabis capsiformis $\mathbf{a}-\mathbf{b}$ dorsal view $\mathbf{c}$ lateral view; $\mathbf{d}-\mathbf{f}$ male genitalia: $\mathbf{d}$ pygophore $\mathbf{e}$ paramere $\mathbf{f}$ aedeagus $\mathbf{g}-\mathbf{j}$ female genitalia: $\mathbf{g}-\mathbf{h}$ genital segment $\mathbf{i}$ first gonapophysis and gonocoxite $1 \mathbf{j}$ second gonapophysis and gonocoxite 2. (apb: articulatory apparatus; ds: ductus seminis; en: endosoma; fa: anterior fibula; fbe: external fibula; ga1 and ga2: gonapophysis 1 and 2; gm: gonangulum; gx1 and 2: gonocoxites 1 and 2, pa: paramere, ptgIX: paratergite IX ; sty: styloid; v: vagina). Figures a-c scale line $1 \mathrm{~mm} ; \mathbf{d}-\mathbf{j}$ scale line $0.2 \mathrm{~mm}$.

Distribution outside Argentina. Brazil: Santaren, Corumbá, Rio de Janeiro; Chile: Arica, Continental Chile and Easter Island; Guyana; México; Peru: Lima; Uruguay: Montevideo.

According to Kerzhner (2007) this species is widely distributed in nearly all tropi$\mathrm{cal}$ and subtropical regions of the world, the Americas from the USA to Argentina.

Measurements. Male $(n=5)$ : Length 8.54-9.10 (mean = 8.74). Head: length $0.92-1.06($ mean $=1.00)$, width $0.71-0.72($ mean $=0.712)$; eye width $0.28-0.33$ (mean $=0.31)$, interocular width $0.28-0.32($ mean $=0.30)$. Rostrum: ratio of segment lengths about $1: 2.59: 2.73: 1.38$. Antenna: ratio of segment about $1: 1.75: 1.76$ : 1.07. Pronotum length $1.19-1.35$ (mean $=1.25)$, width $1.27-1.42$ (mean $=1.36)$. Hemelytra length 6.10-6.60 $($ mean $=6.38)$. Abdomen: length 3.05-3.76 $($ mean $=$ $3.38)$, width 1.14-1.70 (mean $=1.45)$. Legs: fore femora: length $2.13-2.16$ (mean $=2.14)$, width $0.31-0.36($ mean $=0.34)$; middle femora: length $1.87-1.98($ mean $=$ $1.95)$, width $0.12-0.23$ (mean $=0.16)$; hind femora $3.05-3.26($ mean $=3.13)$, width $0.12-0.15($ mean $=0.13)$. Fore tibiae: length $1.70-1.86($ mean $=1.79)$, width 0.10 ; 
middle tibiae: length $1.77-1.92($ mean $=1.84)$, width $0.05-0.07($ mean $=0.064)$; hind tibiae: length 3.48-3.76 (mean $=3.66)$, width 0.05-0.07 $($ mean $=0.064)$.

Female $(\mathrm{n}=5)$ : Length 7.77-9.87 $($ mean $=8.85)$. Head: length $0.98-1.13($ mean $=$ 1.03 ), width $0.71-0.78$ (mean $=0.73)$; eye width $0.31-0.35$ (mean $=0.33)$, interocular width $0.28-0.32$ (mean $=0.30)$. Rostrum: ratio of segment lengths about $1: 2.42: 2.57$ : 1.28. Antenna: ratio of segment about $1: 1.69: 1.73: 1.07$. Pronotum length $1.27-1.42$ $($ mean $=1.34)$, width 1.42-1.63 $($ mean $=1.51)$. Hemelytra length 5.53-7.17 $($ mean $=6.44)$. Abdomen: length 3.12-3.69 (mean = 3.46), width 1.04-1.56 (mean = 1.32). Legs: fore femora: length 2.08-2.27 (mean $=2.23$ ), width $0.35-0.39$ (mean $=0.37)$; middle femora: length 1.91-2.13 (mean = 2.03), width 0.20-0.28 (mean = 0.22); hind femora 3.12-3.33 (mean $=3.2)$, width $0.12-0.26($ mean $=0.19)$. Fore tibiae: length $1.82-1.91($ mean $=1.86)$, width $0.10-0.12($ mean $=0.104)$; middle tibiae: length $1.91-2.05($ mean $=1.97)$, width $0.07-0.12$ (mean $=0.08)$; hind tibiae: length 3.74-3.97 (mean = 3.85), width 0.07.

Description. General coloration light brown. Body elongated, covered with white setae over the surface. Head with whitish pilosity and sparse long setae, more abundant ventrally; brown area between eyes and antennae and post-ocular region laterally. Rostrum passing fore coxae, segment IV brown distally. Antennae long and slender with setae. Pronotum pilose with a brown stripe in the middle (in some specimens diffused); anterior lobe tinged with brown; posterior lobe with a suture and granulate. Scutellum brown in the centre and with two depressions, and the sides clear. In some specimens meso- and metasternum dark brown. Pro- meso- and metapleura and abdomen ventral sides with a brown stripe. Abdomen with abundant sparse setae, not uniformly pigmented, connexivum without spots. Legs long and slender, with long white setae.

Biology. Ojeda-Peña (1971) and Cornelis et al. (2012) described the nymphs, eggs, and biology. The last authors collected the material using a sweeping net in Medicago sativa L. (Fabaceae).

\section{Nabis seticrus Harris, 1930}

Nabis seticrus Harris 1930. Annals of the Carnegie Museum, 19:241-248. Volpi and Coscarón 2010. Zootaxa 2513: 59.

Distribution in Argentina. Salta

Distribution outside Argentina. Brazil: Chapada, Rio de Janeiro.

\section{Nabis roripes Stål, 1860}

http://species-id.net/wiki/Nabis_roripes

Nabis roripes Stål 1860. Kongliga Svenska Vetenskaps-Akademiens Handlingar 2: 70. Holotype (NHRS). Reuter 1890. Revue d'Entomologie 9: 297. Reuter 1908. Mémoires de la Société Entomologique de Belgique 15: 99-101. Harris 1930. Annals 
of the Carnegie Museum 19: 246. Harris 1939. Notas del Museo de La Plata 26: 376. Volpi and Coscarón 2010. Zootaxa 2513: 59.

Holotype: http://www2.nrm.se/en/het_nrm/r/nabis_roripes.html

Distribution in Argentina. Misiones: Loreto.

Distribution outside Argentina. Brazil: Chapada; Colombia: Pandi (Cundimarca); Peru: San Juan.

\section{Punctipennis complex}

The punctipennis complex (according to Harris (1939)) is comprised of Nabis argentinus Meyer-Dür, Nabis faminei Stål, Nabis paranensis Harris, and Nabis punctipennis Blanchard.

Body with abundant long whitish setae. Head brown; with a line in the middle (widened anteriorly), sides of head and ventrally dark brown. Rostrum pallid testaceus, segment I darker at base, IV distally darker. Pronotum with a dark brown stripe in the middle, anterior lobe with irregular fuscous patterns. Scutellum black with a yellowish spot at the sides of the base; with two fovea in the center the area between noticeably depressed. Abdomen above and a wide line on each side sordid brown. Legs pilose, mottled.

\section{Nabis paranensis Harris, 1931}

http://species-id.net/wiki/Nabis_paranensis

Figs $7 \mathrm{a}-\mathrm{i}, 11 \mathrm{a}, 12 \mathrm{a}, 13 \mathrm{~d}$

Nabis paranensis Harris 1931. Annals of the Zoological Museum of Polonian 9: 182. Harris 1939. Notas del Museo de La Plata 26: 374. Volpi and Coscarón 2010. Zootaxa 2513: 58 .

Material examined. BUENOS AIRES: 1 ô (MLP); José C. Paz 34³0'54.38"S; $58^{\circ} 45^{\prime} 58.49 " \mathrm{~W}, 1940$ J. A. Rosas Costa leg. Col., Harris det., 1 o (MLP); La Plata

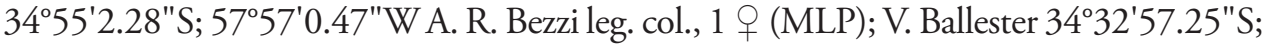

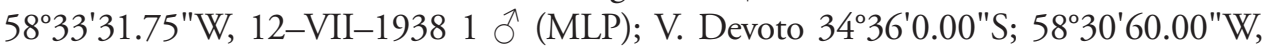

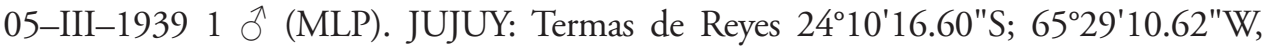

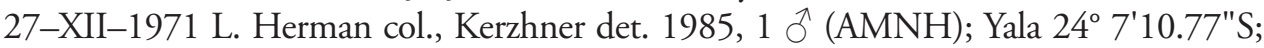
6524'14.34"W, 12-III-1939 Biraben-Scott leg. col., 1 ${ }^{\Uparrow}, 2$ 우 (MLP). LA PAMPA: E. Castex 3554'49.15"S; 64¹7'19.94"W, 31-I-1957 Torres-Ronderos col., 2 우우 (MLP); Parque Luro 36 57'34.32"S; 64'15'7.15"W, 26-I-1957 Torres-Ronderos col., 1 + (MLP). MISIONES: Delta Paraná Guazú 29-II-1919, Bosq col., 1 ô (MLP).

Distribution in Argentina. Buenos Aires: José C. Paz, La Plata, V. Ballester, V. Devoto; Jujuy: Termas de Reyes, Yala; La Pampa: Eduardo Castex, Parque Luro; Misiones: Delta Paraná Guazú. 


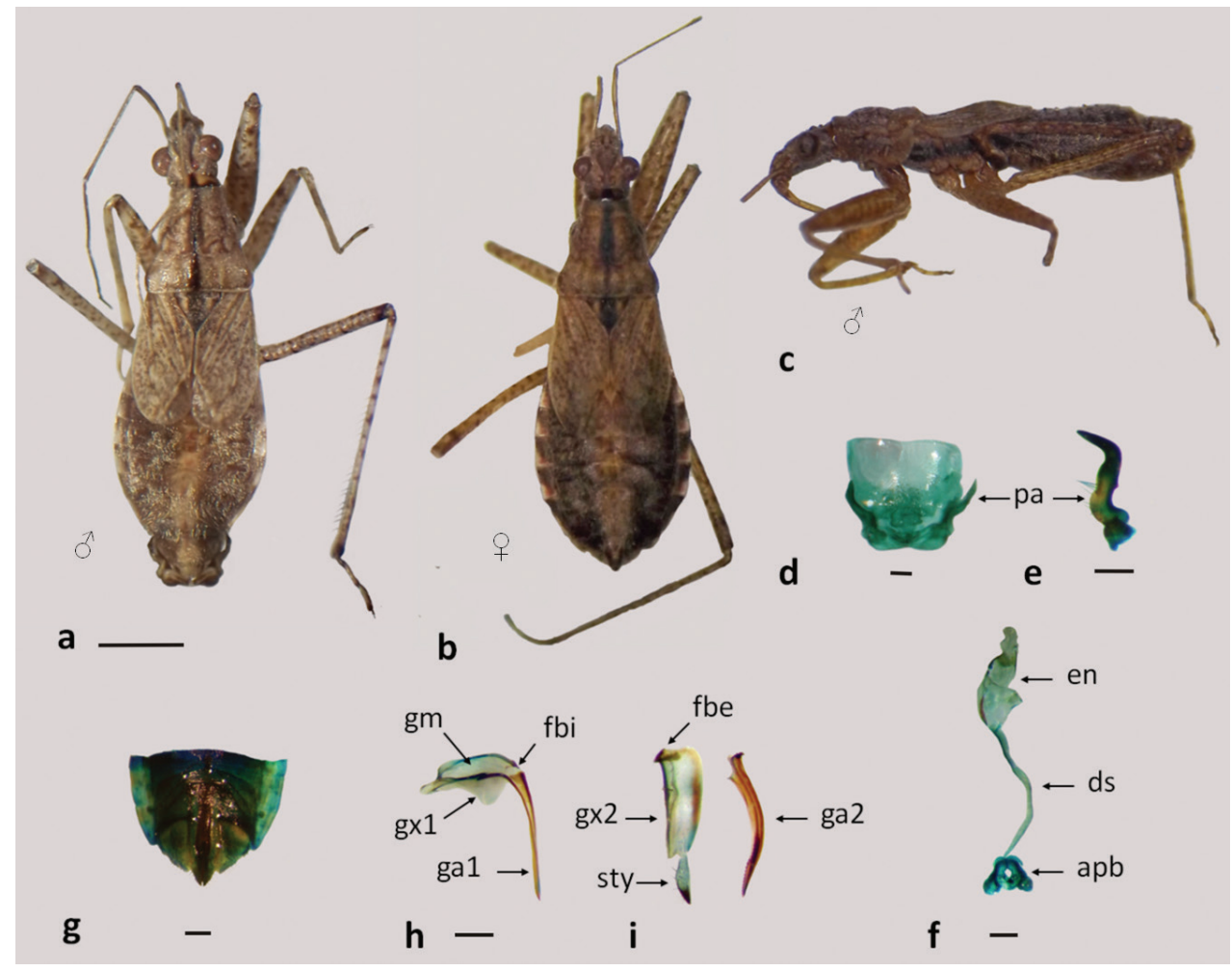

Figure 7. Nabis paranensis $\mathbf{a}-\mathbf{b}$ dorsal view $\mathbf{c}$ lateral view $\mathbf{d}-\mathbf{f}$ male genitalia: $\mathbf{d}$ pygophore e paramere $\mathbf{f}$ aedeagus $\mathbf{g}-\mathbf{i}$ female genitalia: $\mathbf{g}$ genital segment $\mathbf{h}$ first gonapophysis and gonocoxite $1 \mathbf{i}$ second gonapophysis and gonocoxite 2. (apb: articulatory apparatus; ds: ductus seminis; en: endosoma; fbe: external fibula; fbi: internal fibula; ga1 and ga2: gonapophysis 1 and 2; gm: gonangulum; gx1 and 2: gonocoxites 1 and 2, pa: paramere; sty: styloid). Figures a-c scale line $1 \mathrm{~mm} ; \mathbf{d}-\mathbf{i}$ scale line $0.2 \mathrm{~mm}$.

Distribution outside Argentina. Brazil: Parana (São Pedro de Mallet).

New record. Argentina: La Pampa, Winifreda: 36²18'45.30"S; 64 $11^{\prime} 55.45^{\prime \prime W}$, Cornelis col.

Measurements. Male $(\mathrm{n}=5)$ : Length $5.17-6.30$ (mean $=5.53)$. Head: length $0.79-1.00$ (mean $=0.89)$, width $0.79-0.85($ mean $=0.81)$; eye width $0.32-0.42$ (mean $=0.35$ ), interocular width $0.33-0.35$ (mean $=0.33$ ). Rostrum: ratio of segment lengths about $1: 2.28: 1.97: 1.11$. Antenna: ratio of segment about $1: 1.59: 1.32: 1.09$. Pronotum length $1.09-1.24($ mean $=1.14)$, width $1.12-1.40($ mean $=1.22)$. Hemelytra length 1.16-3.94 $($ mean $=1.91)$. Abdomen: length 2.21-3.50 $($ mean $=2.83)$, width 1.46-2.48 $($ mean $=1.71)$. Legs: fore femora: length 1.61-1.98 $($ mean $=1.74)$, width $0.35-0.41$ (mean $=0.38)$; middle femora: length $1.42-1.63($ mean $=1.53)$, width $0.26-0.30($ mean $=0.27)$; hind femora 2.06-2.48 (mean = 2.22), width 0.14-0.18 $($ mean $=0.17)$. Fore tibiae: length $1.31-1.61($ mean $=1.41)$, width $0.10-0.11$ (mean $=0.108)$; middle tibiae: length $1.35-1.65($ mean $=1.45)$, width $0.07-0.11($ mean $=$ $0.08)$; hind tibiae: length $2.33-2.98($ mean $=2.57)$, width $0.07-010($ mean $=0.076)$. 
Female $(\mathrm{n}=5)$ : Length 5.45-5.89 (mean $=5.67)$. Head: length 0.75-1.00 (mean $=0.88)$, width $0.82-0.86($ mean $=0.84)$; eye width $0.35-0.39($ mean $=0.37)$, in terocular width $0.33-0.37($ mean $=0.35)$. Rostrum: ratio of segment lengths about $1: 2.23: 2.41: 1.17$. Antenna: ratio of segment about $1: 1.51: 1.30: 1.03$. Pronotum length $1.12-1.24($ mean $=1.17)$, width $1.20-1.35($ mean $=1.28)$. Hemelytra length 1.50-2.10 $($ mean $=1.79)$. Abdomen: length 2.70-3.00 $($ mean $=2.84)$, width 1.69-1.95 (mean $=1.84)$. Legs: fore femora: length 1.61-2.06 (mean $=1.87)$, width $0.33-0.43$ (mean $=0.39)$; middle femora: length $1.70-2.05($ mean $=1.84)$, width $0.22-0.26($ mean $=0.24)$; hind femora $1.80-2.63($ mean $=2.26)$, width $0.15-0.18$ $($ mean $=0.17)$. Fore tibiae: length $1.27-1.61($ mean $=1.49)$, width $0.09-0.11($ mean $=$ $0.10)$; middle tibiae: length $1.39-1.80($ mean $=1.60)$, width $0.07-0.11($ mean $=0.08)$; hind tibiae: length $2.63-3.15$ (mean $=2.78$ ), width 0.07 .

Description. Eyes prominent; antennae and legs longer than in the others species of the complex. Length of first antennal segment equal or slightly shorter than the distance between the eyes. Length of second antennal segment subequal to the width of the base of the pronotum. In brachypterous forms, hemelytra reaching to the fifth abdominal segment and membrane slightly surpassing the apex of the corium. Paramere distally shorter and more thickened than in $N$. faminei and $N$. punctipennis. Paramere with a thickening on the inner margin of the blade and a protuberance on the outer margin of the blade. Female genitalia with the styloid constricted basally and sharp distally.

\section{Nabis argentinus Meyer-Dür, 1870}

http://species-id.net/wiki/Nabis_argentinus

Figures $8 \mathrm{a}-\mathrm{i}, 11 \mathrm{~b}, 12 \mathrm{~b}, 13 \mathrm{~d}$

Nabis argentinus Meyer-Dür 1870. Mitteilungen der Schweizerischen Entomologischen Gesellschaft 3: 177. Harris 1939. Notas del Museo de La Plata 26: 373. Ruffinelli and Pirán 1959. Boletín de la Facultad de Agronomía de Montevideo 51: 40. Volpi and Coscarón 2010. Zootaxa 2513: 58.

Coriscus argentinus Stål 1873. Köngliga Svenska Vetenskaps-Akademiens Handlingar 11: 114.

Nabis punctipennis: Berg 1879. Anales de la Sociedad Científica Argentina 9: 143. Berg 1892. Anales de la Sociedad Científica Argentina 34: 104. Pennington 1920. Lista de los Hemípteros Heterópteros de la República Argentina. Segunda Parte: 26.

Material examined. BUENOS AIRES: 1852 Harris det., 1 q (MLP), 15-I-1921 1 (MLP), XII-1938 Drake and Richardson col., Harris det., 1 ภ, 1 ㅇ (MLP); Bahía Blanca 3842'42.04"S; 62¹6'5.08"W, III-1984 Mianzan col., 3 우 (MLP), 18-II-1977 S.

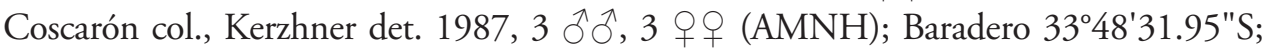
59 30'16.86"W, 1 ○े (MLP), Harris det. 1 o (MLP); Ciudad de Buenos Aires 343'으.30"S; 58²2'23.38"W, 24-XII-1918 Bosq col., 1 q (MLP), 25-XII-1918 1 ㅇ (MLP), 29-I-1919 Bosq col., 1 ○े (MLP), 29-III-1919 Bosq col., 1 q (MLP); 


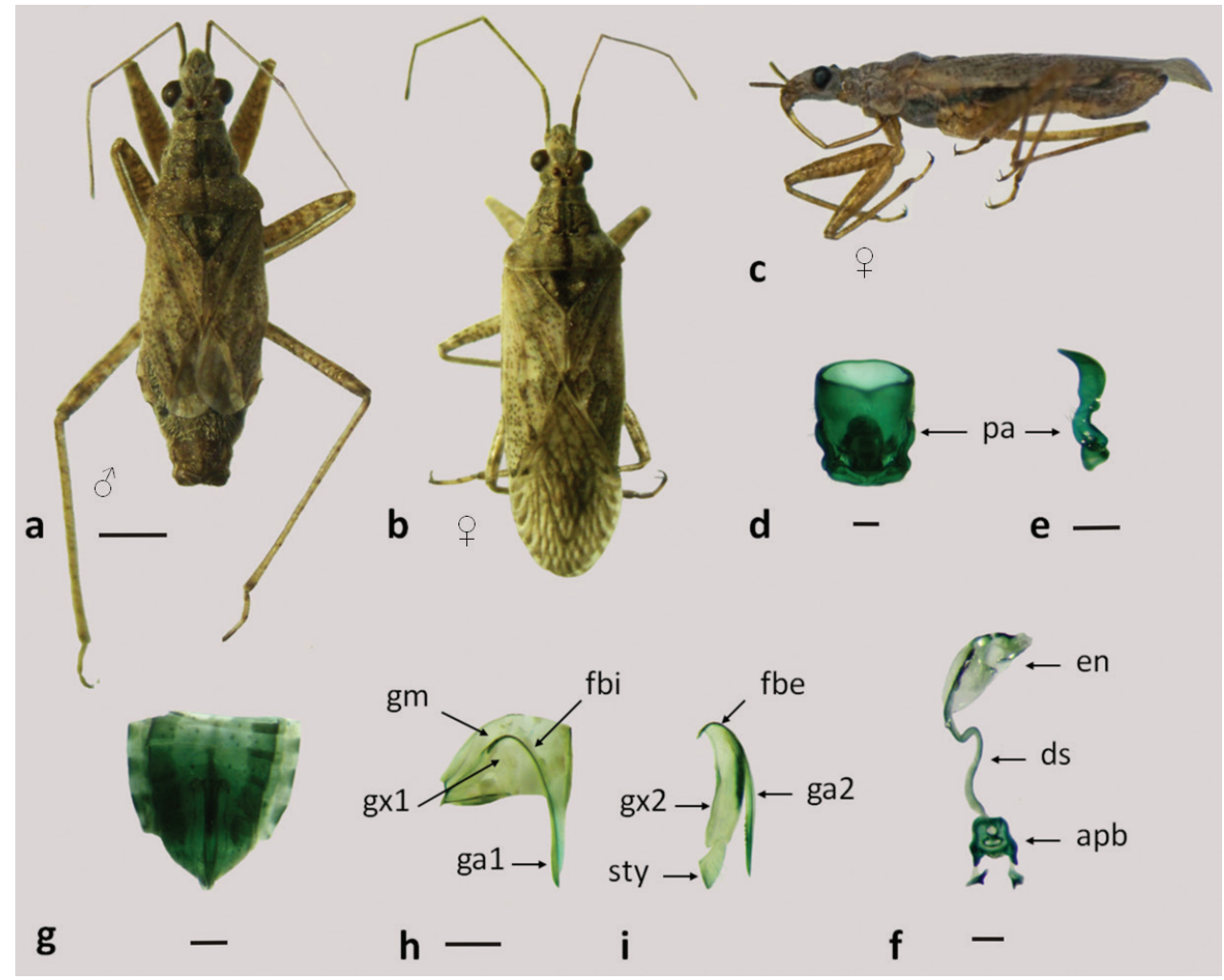

Figure 8. Nabis argentinus $\mathbf{a}-\mathbf{b}$ dorsal view $\mathbf{c}$ lateral view $\mathbf{d}-\mathbf{f}$ male genitalia: $\mathbf{d}$ pygophore e paramere $\mathbf{f}$ aedeagus $\mathbf{g}-\mathbf{i}$ female genitalia: $\mathbf{g}$ genital segment $\mathbf{h}$ first gonapophysis and gonocoxite $1 \mathbf{i}$ second gonapophysis and gonocoxite 2. (apb: articulatory apparatus; ds: ductus seminis; en: endosoma; fbe: external fibula; fbi: internal fibula; ga1 and ga2: gonapophysis 1 and 2; gm: gonangulum; gx1 and 2: gonocoxites 1 and 2, pa: paramere; sty: styloid). Figures a-c scale line $1 \mathrm{~mm} ; \mathbf{d}-\mathbf{i}$ scale line $0.2 \mathrm{~mm}$.

Flores 34³7'60.00"S; 58²8'0.00"W, 7-III-1917 Bosq col., 1 ㅇ (MLP); José C. Paz 34²30'54.38"S; 5845'58.49"W, XII-1938 1 ○े (MLP), 07-III-1939 1 ㅇ (MLP), 21-I19391 đ̊ (MLP), 05-IX-1939, 1 \& (MLP), 1940 J. A. Rosas Costa leg. col.; La Colina

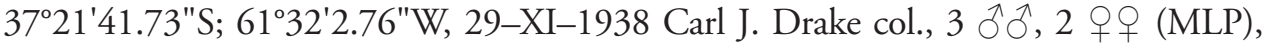
29-XI-1938 Carl J. Drake col., Harris det. 1 o (MLP); La Madrid 37¹4'51.31"S;

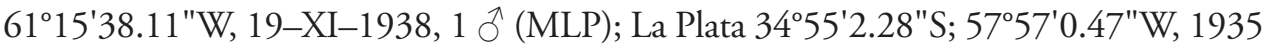
J. A. Rosas Costa col., Harris det., 1 q (MLP); Harris det., 1 §ิ, 1 \& (MLP), A. R. Bezzi leg. col., 1 \& (MLP), 03-III-2003 P. M. Dellape col., 1 q (MLP); Monte Hermoso $38^{\circ} 58^{\prime} 58.01 " \mathrm{~S}$; 6117'50.69"W, 28-II-1957 Torres-Ronderos col., 1 \& (MLP); Pedro Luro 39³0'5.86"S; 62²1'0.10"W, 08-II-1941 Biraben col., 3 우우 (MLP); Punta del

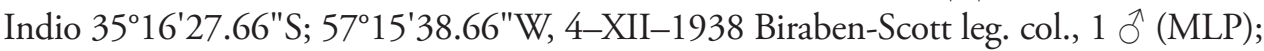

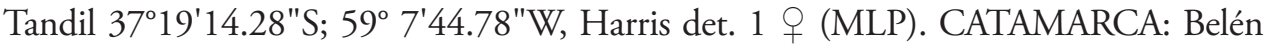
273' 59.42"S; 67 1'59.09"W, 02-III-1939 Biraben-Scott leg col. 2 우 (MLP). CÓRDOBA: Alta Gracia 31³9'16.39"S; 64²5'50.17"W, I-1935 C. Bruch leg. Col., Harris 


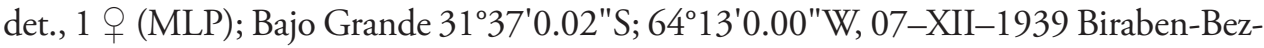

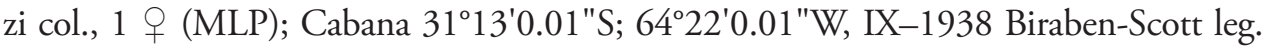
col., 1 (MLP), 10-II-1942 Biraben col., 1 ㅇ (MLP), 10-XI-1942 Biraben col., $1 \hat{\jmath}$

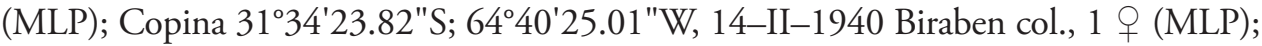
La Puerta 3053'38.28"S; 63¹5'7.24"W, 23-I-1940 Biraben col., 1 q (MLP); Mar Chiquita 3047'60.00"S; 62 52'59.99"W, 22-I-1940 Biraben col., 1 ㅇ (MLP); Marull 30 $59^{\prime} 45.16 " S$; 62 49'37.61"W, 22-I-1940 Biraben col., 2 우 (MLP); San Antonio

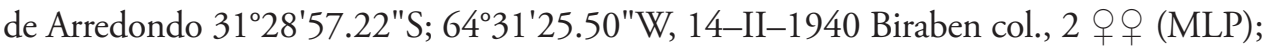

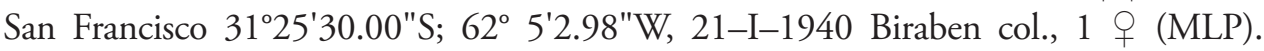
CHACO: Nueva Pompeya 245'53.10"S; 6128'59.70"W, Harris det., 1 ㅇ (MLP). ENTRE RÍOS: Gualeguaychú 330'54.06"S; 58³1'9.28"W, 20-XII-1941 Biraben-

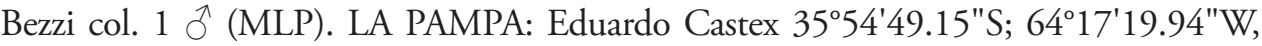

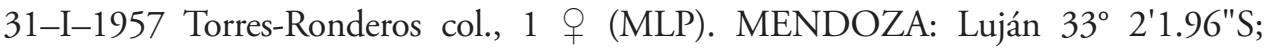

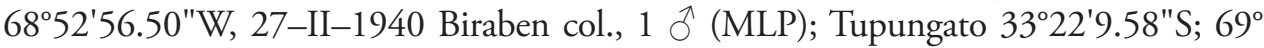
8'43.59"W, 27-II-1940 Biraben col., 1 \& (MLP). RÍO NEGRO: I-1951 1 q (MLP); San Antonio Oeste 40 43'49.84"S; 64 $56^{\prime} 57.03^{\prime \prime W}, 20-I I-1915,2$ 우 (MLP). SALTA: San Lorenzo 2443'46.97"S; 65²9'7.42"W, 14-VII-1939 1 9 (MLP). SAN LUIS: Nogolí 32 $55^{\prime} 6.09^{\prime \prime S}$; 66 19'30.79"W, 21-II-1940 Biraben col., $10^{\top}, 1$ + (MLP); Quines 32 14'1.21"S; 6548' 8.50"W, 18-II-1940 Biraben col., 1 q (MLP); San Francisco 3316'51.13"S; 66¹8'32.36"W, 20-II-1940 Biraben col., 1 + (MLP).

Distribution in Argentina. Buenos Aires: Bahía Blanca, Baradero, Ciudad de Buenos Aires, Flores, José C. Paz, La Colina, La Madrid, La Plata, Monte Hermoso, Pedro Luro, Punta del Indio; Catamarca: Belén; Córdoba: Alta Gracia, Bajo Grande, Cabana, Copina, La Puerta, Mar Chiquita, Marull, San Antonio de Arredondo, San Francisco; Chaco: Nueva Pompeya; Entre Ríos: Gualeguaychú; La Pampa: Eduardo Castex; Mendoza: Luján, Tupungato; Río Negro: San Antonio Oeste; Salta: San Lorenzo; San Luis: Nogolí, Quines, San Francisco.

Distribution outside Argentina. Uruguay: Artigas, Canelones, Colonia, Durazno, Maldonado, Montevideo, Paysandú.

New record. Argentina: La Pampa, Winifreda: 36²18'45.30"S; 64 $11^{\prime} 55.45^{\prime \prime W}$, Cornelis col.

Measurements. Male $(n=5)$ : Length 5.32-6.81 (mean = 6.34). Head: length $0.78-0.99($ mean $=0.86)$, width $0.85-0.92($ mean $=0.86)$; eye width $0.38-0.42$ (mean $=0.40)$, interocular width $0.38-0.42($ mean $=0.41)$. Rostrum: ratio of segment lengths about $1: 2.54: 2.48: 1.29$. Antenna: ratio of segment about $1: 1.73: 1.33: 0.90$. Pronotum length $1.06-1.27$ (mean $=1.17)$, width $1.42-1.77($ mean $=1.57)$. Hemelytra length 2.48-4.68 $($ mean $=4.11)$. Abdomen: length 1.91-2.69 $($ mean $=2.28)$, width $1.42-1.77($ mean $=1.65)$. Legs: fore femora: length 1.70-1.77 $($ mean $=1.73)$, width $0.35-0.42$ (mean $=0.37)$; middle femora: length $1.42-2.05($ mean $=1.67)$, width $0.28-0.32($ mean $=0.29)$; hind femora $2.27-2.34($ mean $=2.29)$, width $0.14-0.21$ $($ mean $=0.18)$. Fore tibiae: length $1.42-1.49($ mean $=1.43)$, width $0.10-0.12($ mean $=0.105)$; middle tibiae: length $1.49-1.60($ mean $=1.52)$, width $0.07-0.10($ mean $=$ $0.09)$; hind tibiae: length $2.55-2.84($ mean $=2.70)$, width $0.07-010($ mean $=0.08)$. 
Female $(\mathrm{n}=5)$ : Length 5.75-7.10 $($ mean $=6.57)$. Head: length $0.88-1.06($ mean $=$ $0.98)$, width $0.74-0.92($ mean $=0.88)$; eye width $0.38-0.42($ mean $=0.41)$, interocular width $0.35-0.42($ mean $=0.39)$. Rostrum: ratio of segment lengths about $1: 2.67: 2.58$ : 1.35. Antenna: ratio of segment about $1: 1.82: 1.41: 0.91$. Pronotum length 1.13-1.35 $($ mean $=1.25)$, width 1.13-1.84 $($ mean $=1.65)$. Hemelytra length $1.60-4.97($ mean $=$ 4.25). Abdomen: length 2.69-3.05 ( $\mathrm{mean}=2.88)$, width $1.77-2.13$ (mean = 1.88). Legs: fore femora: length $1.70-1.91($ mean $=1.80)$, width $0.35-0.42($ mean $=0.37)$; middle femora: length 1.60-1.77 (mean = 1.66), width 0.28-0.32 $($ mean $=0.29)$; hind femora $2.27-2.48($ mean $=2.40)$, width $0.15-0.21($ mean $=0.19)$. Fore tibiae: length $1.45-1.52$ $($ mean $=1.48)$, width $0.10-0.14($ mean $=0.12)$; middle tibiae: length $1.55-1.63($ mean $=$ $1.60)$, width 0.10 ; hind tibiae: length 2.84-3.05 (mean $=2.89)$, width 0.10 .

Description. Pronotum in macropterous form greatly expanded behind, a third wider than long; posterior lobe markedly arched upward. Pronotum in brachypterous form visibly wider than long, but strongly compressed on the sides between the anterior and posterior lobes. Hemelytra in macropterous forms surpassing apex of abdomen; in brachypterous forms, reaching to the base of sixth abdominal segment. Paramere with the distal area more thickened than in the other species of the complex; the blade wide, with a protuberance on the outer margin. Base of the paramere more constricted than in N. paranensis. Female genitalia with the styloid more thickened than in $N$. paranensis.

\section{Nabis faminei Stål, 1859}

http://species-id.net/wiki/Nabis_faminei

Figs $9 \mathrm{a}-\mathrm{i}, 11 \mathrm{c}, 12 \mathrm{c} 13 \mathrm{~d}$

Nabis faminei Stål 1859. Kongliga Svenska Fregattens Eugenies Resa Omkring Jorden 4: 260. Lectotype (NHRS). Reuter 1872. Öfversigt af Kongliga Svenska Vetenskaps-Akademiens Forhandlingar 29: 92. Berg 1879. Anales de la Sociedad Científica Argentina: 145. Pennington 1921. Lista de los Hemípteros de la República Argentina. Segunda parte: 26. Harris 1939. Notas del Museo de La Plata 26: 372. Prado 2008. Boletín del Museo Nacional de Historia Natural Chile 57: 44. Volpi and Coscarón 2010. Zootaxa 2513: 58.

Nabis punctipennis: Reuter 1908. Mémoires de la Société Entomologique de Belgique 15: 122.

Lectotype: http://www2.nrm.se/en/het_nrm/f/nabis_faminei.html

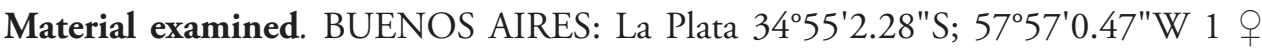
(MLP). TIERRA DEL FUEGO: Harris det., 1 + (MLP); Cabana Ruby 17-I-1988, 1

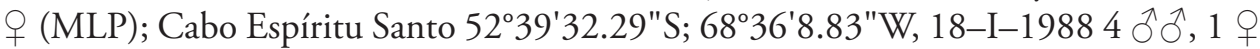
(MLP); Estancia La Indiana 54²0'46.48"S; 67²5'44.27"W, 17-I-1988 2 ふぇ, 1 우 (MLP); Lago Fagnano 17-I-1988 Leg. Molta and Lombardo col., 2 우 (MLP); Paso Garibaldi 54²41'29.80"S; 6751'0.99"W, 17-I-1988 (MLP); Río Chico, Las Violetas 54ํำ'55.02"S; 68¹8'29.55"W, 13-I-1988 2 우우 (MLP). 


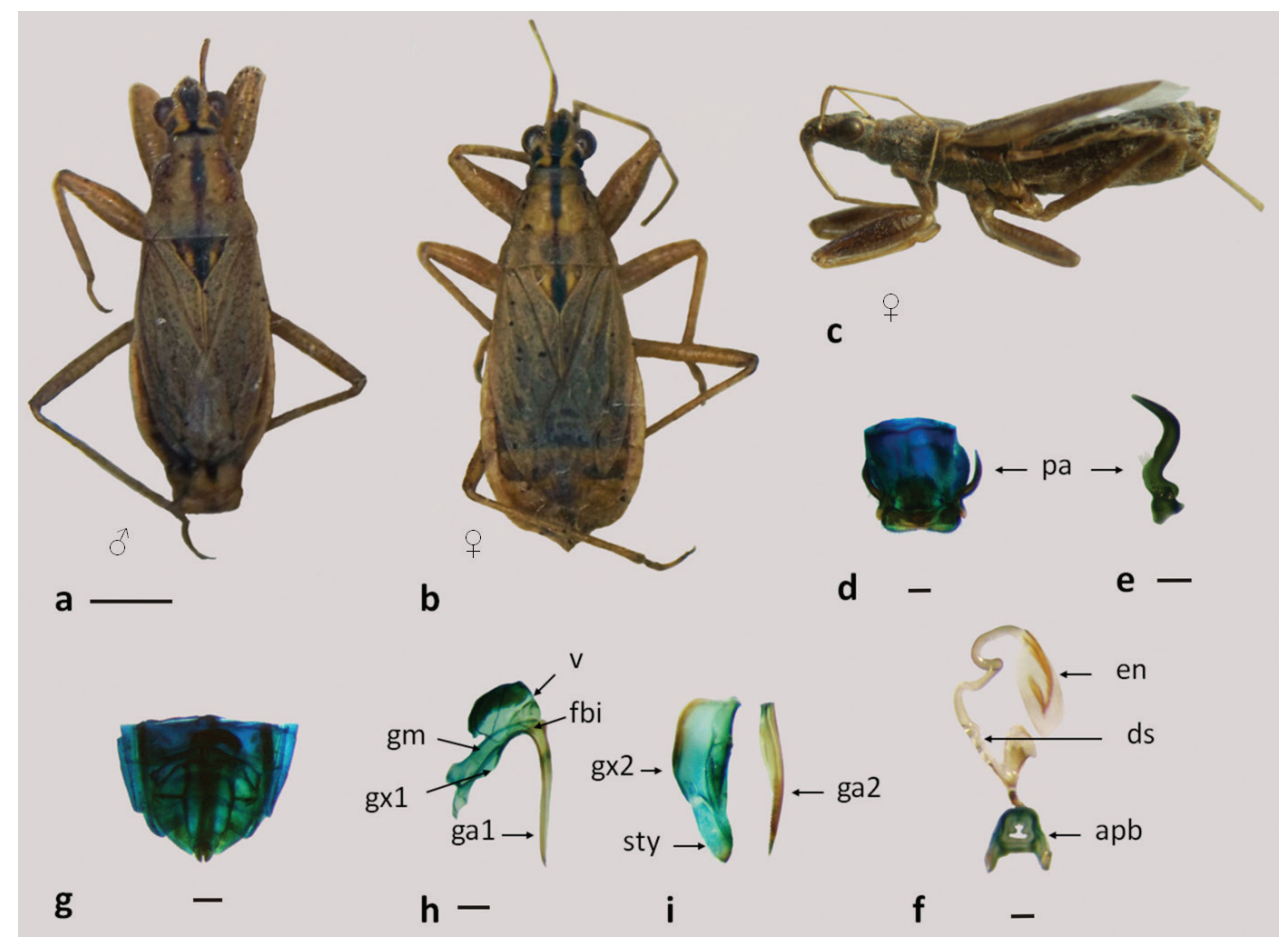

Figure 9. Nabis faminei $\mathbf{a}-\mathbf{b}$ dorsal view $\mathbf{a}$ male $\mathbf{b}$ female $\mathbf{c}$ lateral view $\mathbf{d}-\mathbf{f}$ male genitalia: $\mathbf{d}$ pygophore e paramere $\mathbf{f}$ aedeagus $\mathbf{g}-\mathbf{i}$ female genitalia: $\mathbf{g}$ genital segment $\mathbf{h}$ first gonapophysis and gonocoxite $1 \mathbf{i}$ second gonapophysis and gonocoxite 2. (apb: articulatory apparatus; ds: ductus seminis; en: endosoma; fbi: internal fibula; ga1 and ga2: gonapophysis 1 and 2; gm: gonangulum; gx 1 and 2: gonocoxites 1 and 2, pa: paramere; sty: styloid; v: vagina). Figures a-c scale line $1 \mathrm{~mm}$; $\mathbf{d}-\mathbf{i}$ scale line $0.2 \mathrm{~mm}$.

Distribution in Argentina. Buenos Aires: La Plata; Patagonia, Tierra del Fuego: Cabana Ruby, Cabo Espíritu Santo, Estancia La Indiana, Lago Fagnano, Paso Garibaldi, Río Chico (Las Violetas).

Distribution outside Argentina. Chile.

Measurements. Male $(\mathrm{n}=3)$ : Length 4.92-5.84 (mean $=5.26)$. Head: length $0.82-0.93($ mean $=0.87)$, width $0.82-0.86($ mean $=0.83)$; eye width $0.36-0.37$ (mean $=0.365$ ), interocular width 0.37 . Rostrum: ratio of segment lengths about 1: 2.26 : 0.59 : 0.62. Antenna: ratio of segment about 1: 1.64 (segments III and IV missing). Pronotum length $1.01-1.16($ mean $=1.08)$, width $1.16-1.46($ mean $=1.28)$. Hemelytra length 2.44-3.79 $($ mean $=2.91)$. Abdomen: length 2.17-2.66 $($ mean $=2.48)$, width $1.50-1.67($ mean $=1.57)$. Legs: fore femora: length $1.36-1.39($ mean $=1.38)$, width $0.39-0.41$ (mean $=0.396)$; middle femora: length $1.25-1.27($ mean $=1.265)$, width $0.30-0.33$ (mean $=0.31)$; hind femora $1.78-1.87($ mean $=1.82)$, width $0.17-0.18$ $($ mean $=0.175)$. Fore tibiae: length $1.09-1-15($ mean $=1.12)$, width $0.09-0.12($ mean $=0.10)$; middle tibiae: length $1.06-1.17($ mean $=1.13)$, width $0.075-0.09$ (mean $=$ $0.08)$; hind tibiae: length $2.03-2.14($ mean $=2.08)$, width $0.06-0.07($ mean $=0.065)$. 
Female $(\mathrm{n}=3)$ : Length 5.07-5.84 (mean $=5.39)$. Head: length 0.82-0.93 (mean $=0.88)$, width $0.78-0.86($ mean $=0.82)$; eye width $0.36-0.39($ mean $=0.37)$, in terocular width $0.36-0.37$ (mean $=0.365)$. Rostrum: ratio of segment lengths about $1: 2.19: 2.43: 1.19$. Antenna: ratio of segment about $1: 1.47: 1.41: 1.15$. Pronotum length 1.09-1.20 (mean $=1.13)$, width $1.27-1.42($ mean $=1.32)$. Hemelytra length 2.60-3.12 $($ mean $=2.84)$. Abdomen: length 2.42-2.59 $($ mean $=2.52)$, width $1.80-1.95$ (mean $=1.86)$. Legs: fore femora: length 1.09-1.60 (mean $=1.35)$, width $0.36-0.42($ mean $=0.38)$; middle femora: length 1.21-1.51 $($ mean $=1.33)$, width $0.21-0.30($ mean $=0.26)$; hind femora $1.75-2.24($ mean $=1.94)$, width 0.18 . Fore tibiae: length $1.18-1.33($ mean $=1.24)$, width $0.09-0.12($ mean $=0.11)$; middle tibiae: length $1.15-1.33($ mean $=1.23)$, width $0.09-0.10($ mean $=0.093)$; hind tibiae: length $2.12-2.39($ mean $=2.21)$, width $0.075-0.10($ mean $=0.088)$.

Description. Similar to $N$. punctipennis but with the body more robust, the eyes larger, and the antennae and legs shorter. Length of antennal segment II hardly equal to the width of the head across eyes. Anterior lobe of pronotum arcuate, strongly raised above the collar. Hemelytra exposing part of the abdomen. Legs robust, anterior femora thickened. Paramere blade thin, with apex pointed. Female genitalia with thick and robust styloid.

\section{Nabis punctipennis Blanchard, 1852}

http://species-id.net/wiki/Nabis_punctipennis

Figs 10a-j, 11d, 12d, 13d

Nabis punctipennis Blanchard 1852. In: Gay C (Ed) Historia Física y Política de Chile 7: 161. Signoret 1863. Annales de la Société Entomologique de France 3: 577. Reuter 1872. Öfversigt af Kongliga Svenska Vetenskaps-Akademiens Forhandlingar 29: 90. Harris 1939. Notas del Museo de La Plata 26: 370-371. Prado 2008. Boletín del Museo Nacional de Historia Natural Chile 57: 44. Volpi and Coscarón 2010. Zootaxa 2513: 59.

Nabis parvulus Reuter 1872. Öfversigt af Kongliga Svenska Vetenskaps-Akademiens Forhandlingar 29: 90-91. Synonymized by Reuter 1908: 123.

Coriscus punctipennis Stål 1873. Kongliga Svenska Vetenskaps-Akademiens Handlingar 11: 114 .

Reduviolus punctipennis Reuter 1908. Mémoires de la Société Entomologique de Belgique 15: 122 (in part).

Material examined. CHUBUT: El Hoyo 42 3'51.98"S; 71³1'11.29"W, 21-I-1965 A Kovacs col., Kerzhner det., 19871 đે, 1 9 (AMNH). MENDOZA: Jensen-Haarup det., 1 q (MHND); Chacras de Coria 32 $2^{\circ} 58^{\prime} 51.27^{\prime \prime S}$; 68 ${ }^{\circ} 52^{\prime} 36.84^{\prime \prime W}$ Jensen-Haarup, 1 ㅇ (MHND). NEUQUÉN: Huechulaufquen 3946'4.95"S; 71²2'14.02"W, 23-II-1942

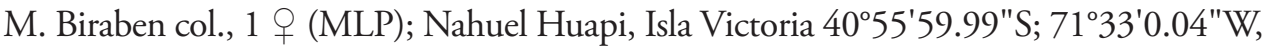

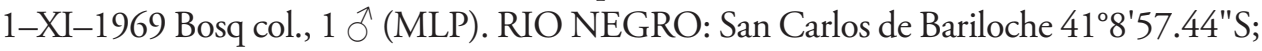

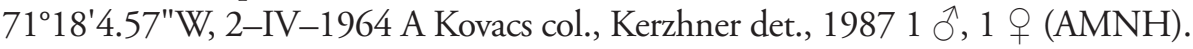




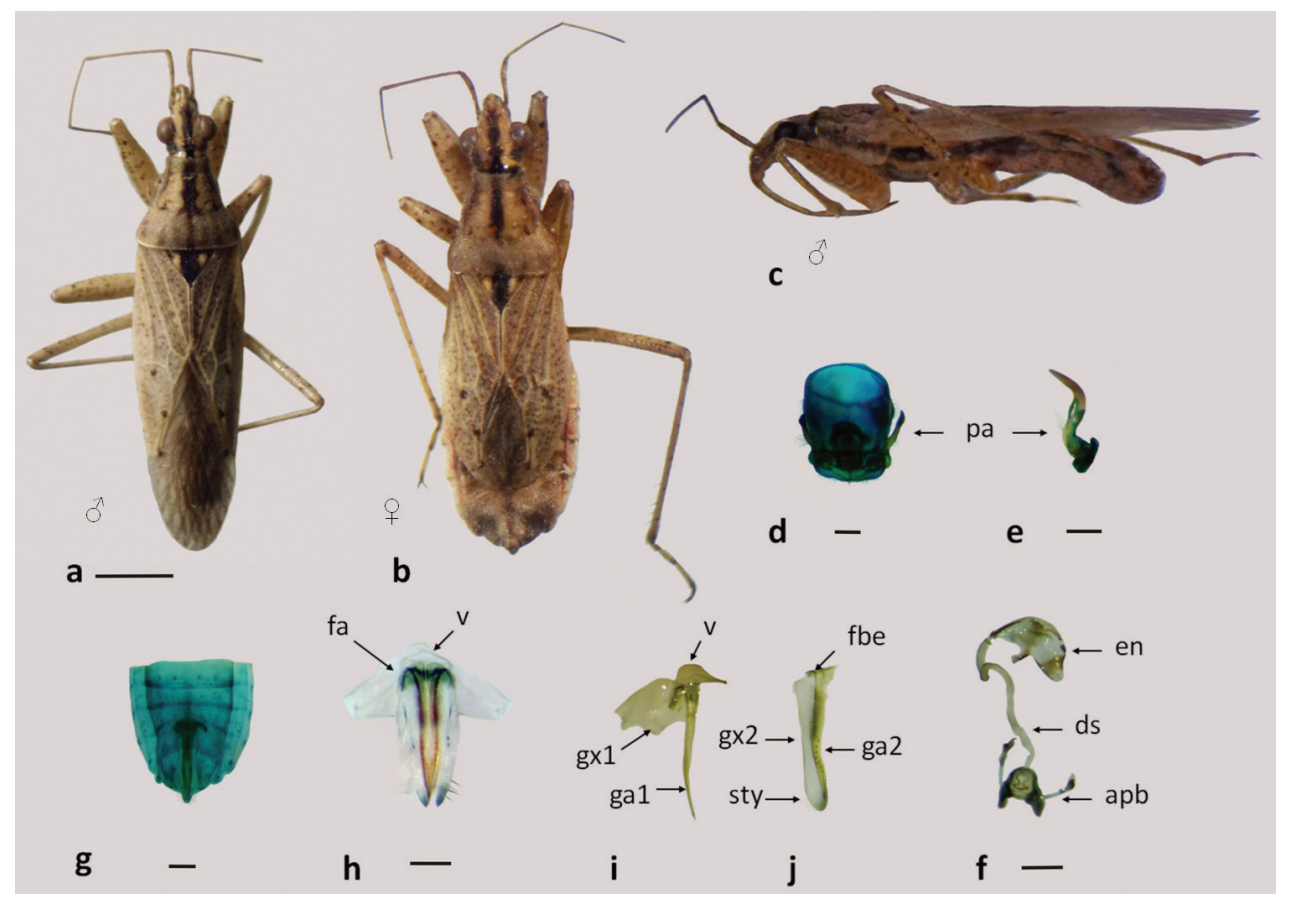

Figure 10. Nabis punctipennis $\mathbf{a}-\mathbf{b}$ dorsal view $\mathbf{c}$ lateral view $\mathbf{d}-\mathbf{f}$ male genitalia: $\mathbf{d}$ pygophore $\mathbf{e}$ paramere $\mathbf{f}$ aedeagus $\mathbf{g}-\mathbf{j}$ female genitalia: $\mathbf{g}-\mathbf{h}$ genital segment $\mathbf{i}$ first gonapophysis and gonocoxite $\mathbf{1} \mathbf{j}$ second gonapophysis and gonocoxite 2. (apb: articulatory apparatus; ds: ductus seminis; en: endosoma; fa: anterior fibula; fbe: external fibula; ga1 and ga2: gonapophysis 1 and 2; gx1 and 2: gonocoxites 1 and 2, pa: paramere; sty: styloid; v: vagina). Figures a-c scale line $1 \mathrm{~mm} ; \mathbf{d}-\mathbf{j}$ scale line $0.2 \mathrm{~mm}$.

Distribution in Argentina. Chubut: El Hoyo; Mendoza: Chacras de Coria; Neuquén: Huechulaufquen, Nahuel Huapi: Isla Victoria; Río Negro: San Carlos de Bariloche.

Distribution outside Argentina. Chile: Colina, Osorno, Puerto Varas. Continental Chile and Archipiélago Juan Fernández.

Measurements. Male $(\mathrm{n}=2)$ : Length 5.26-6.24 (mean = 5.75). Head: length $0.86-0.88($ mean $=0.87)$, width $0.71-0.86($ mean $=0.78)$; eye width $0.33-0.39$ $($ mean $=0.36)$, interocular width $0.37-0.39($ mean $=0.38)$. Rostrum: ratio of segment lengths about $1: 1.91: 2.08: 0.91$. Antenna: ratio of segment about $1: 1.74$ : 1.44: 1.33. Pronotum length $1.16-1.23($ mean $=1.19)$, width $1.27-1.42($ mean $=$ 1.34). Hemelytra length 2.63-4.11 (mean = 3.37). Abdomen: length $2.25-2.41$ $($ mean $=2.33)$, width 1.27-1.39 $($ mean $=1.33)$. Legs: fore femora: length 1.63-1.69 $($ mean $=1.66)$, width $0.35-0.37($ mean $=0.36) ;$ middle femora: length $1.46-1.49$ $($ mean $=1.475)$, width 0.28-0.30 (mean $=0.29)$; hind femora $2.05-2.10$ (mean $=2.07)$, width $0.14-0.15($ mean $=0.145)$. Fore tibiae: length $1.31-1-42($ mean $=$ 1.36 ), width $0.10-0.11$ (mean $=0.105)$; middle tibiae: length 1.35 , width 0.09 $0.10($ mean $=0.095)$; hind tibiae: length $2.36-2.48($ mean $=2.42)$, width $0.07-0.10$ $($ mean $=0.085)$. 


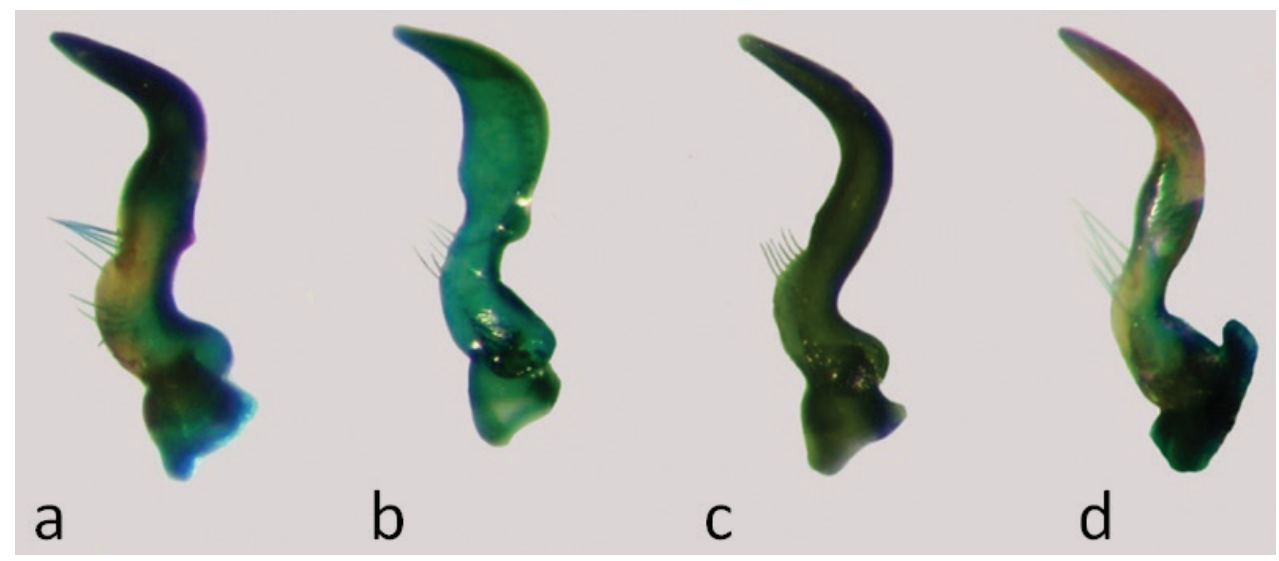

Figure II. Parameres: a $N$. paranensis $\mathbf{b} N$. argentinus $\mathbf{c} N$. faminei $\mathbf{d} N$. punctipennis.

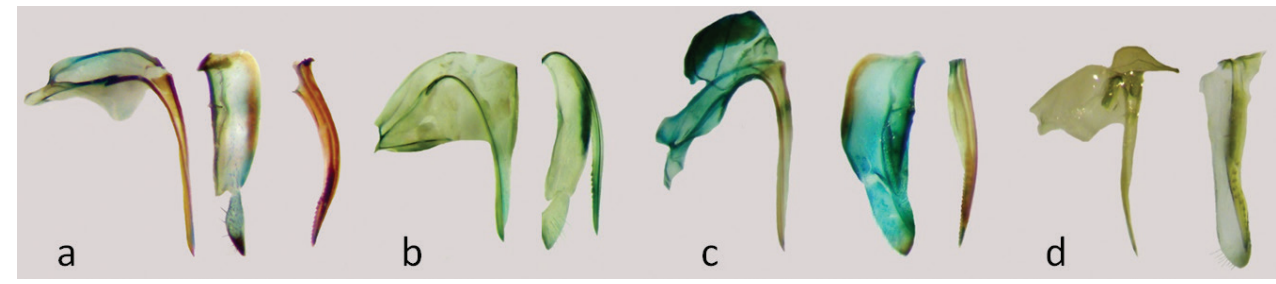

Figure 12. Genital segments of female: a $N$. paranensis $\mathbf{b} N$. argentinus $\mathbf{c} N$. faminei $\mathbf{d} N$. punctipennis.

Female $(\mathrm{n}=5)$ : Length $4.92-6.67$ (mean $=6.06)$. Head: length $0.81-1.06$ (mean $=0.91)$, width $0.78-0.92($ mean $=0.84)$; eye width $0.33-0.42($ mean $=0.37)$, interocular width $0.31-0.40($ mean $=0.35)$. Rostrum: ratio of segment lengths about $1: 2.30: 2.33: 1.24$. Antenna: ratio of segment about $1: 1.61: 1.33: 1.15$. Pronotum length $1.12-1.35($ mean $=1.24)$, width $1.20-1.77($ mean $=1.52)$. Hemelytra length 2.44-4.61 (mean =3.68). Abdomen: length 2.48-3.26 (mean = 2.83), width $1.57-2.13($ mean $=1.78)$. Legs: fore femora: length 1.51-1.61 $($ mean $=1.56)$, width $0.39-0.42($ mean $=0.40)$; middle femora: length $0.45-1.68($ mean $=1.55)$, width $0.26-0.35$ (mean $=0.30)$; hind femora $1.87-2.60($ mean $=2.21)$, width $0.15-0.21$ $($ mean $=0.17)$. Fore tibiae: length 1.31-1.42 $($ mean $=1.35)$, width 0.07-0.11 (mean $=0.10)$; middle tibiae: length 1.27-1.60 $($ mean $=1.42)$, width $0.07-0.11($ mean $=$ $0.09)$; hind tibiae: length 1.81-2.77 $($ mean $=2.40)$, width $0.07-0.10($ mean $=0.08)$.

Description. Length of antennal segment II slightly longer than the width of the head across eyes. Hemelytra in macropterous form surpassing the apex of abdomen, in brachypterous form reaching eight segment. Paramere very similar to $N$. faminei, but with the blade slightly more widened and the base more constricted. Female genitalia with the styloid less thickened than in $N$. faminei. 

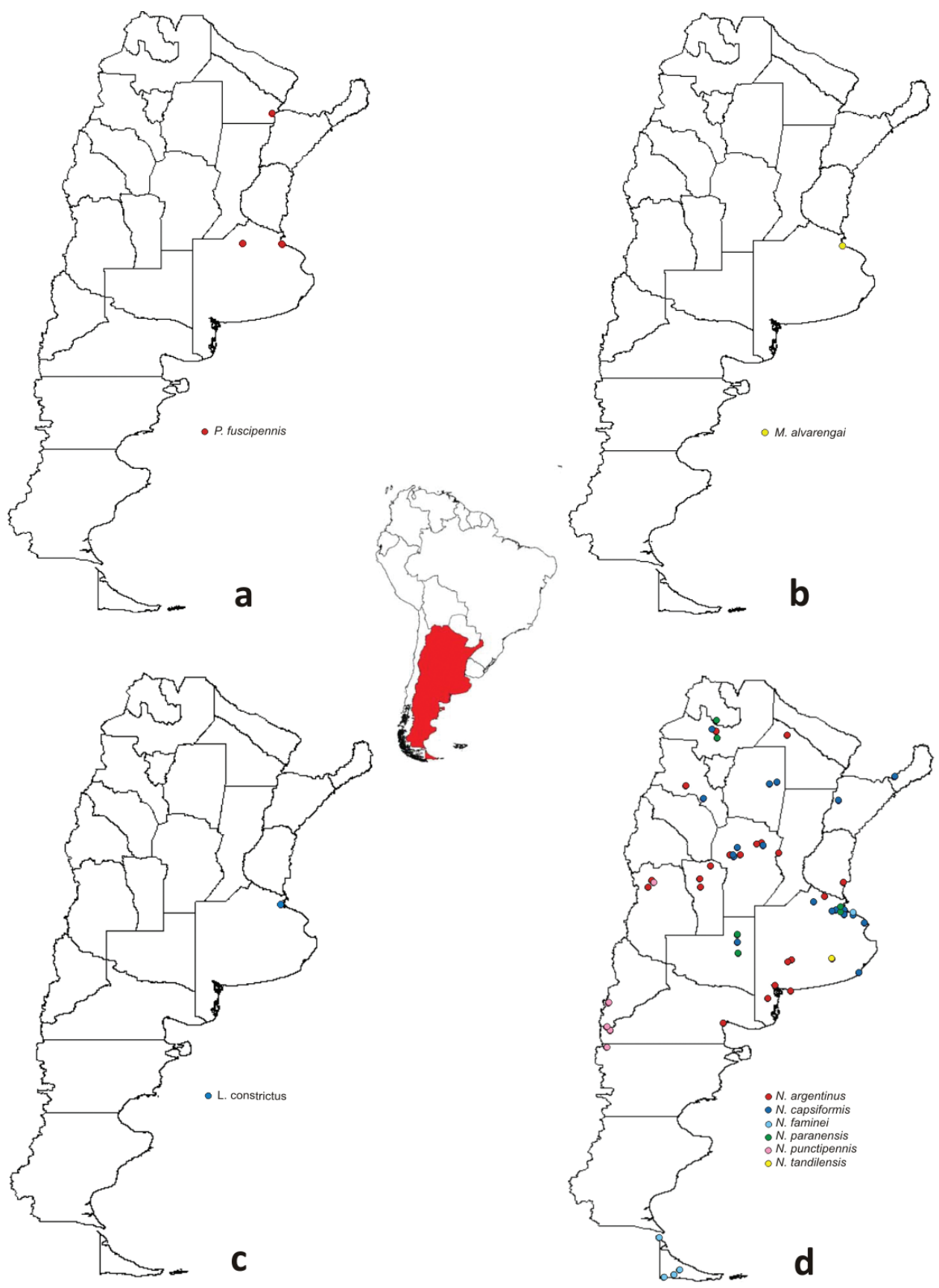

Figure 13. Geographical distribution of species of Nabidae in Argentina: a Pagasa b Metatropiphorus c Lasiomerus d Nabis. 


\section{Acknowledgements}

This work was supported by the Consejo Nacional de Investigaciones Científicas y Técnologicas (CONICET). Special thanks for lending material: Dr. G. Lindberg (Swedish Museum of Natural History); Dr. R. Schuh and Dr. R. Salas (The American Museum of Natural History). We thank Dr. T. Henry (Systematic Entomology Laboratory, USDA, c/o National Museum of Natural History, Smithsonian Institution) and Dr. D. Polhemus (Bishop Museum) for revising and improving the manuscript.

\section{References}

Berg C (1879) Hemiptera Argentina Enumeravit Specisque Novas. Anales de la Sociedad Científica Argentina 9-316.

Berg C (1884) Addenda et emendata ad Hemiptera Argentina. Anales de la Sociedad Científica Argentina 2: 97-118.

Berg C (1892) Nova Hemiptera faunarum Argentinae et Uruguayensis. Anales de la Sociedad Científica Argentina 33: 151-155.

Blanchard E (1852) Order VII: Hemipteros. In: Gay C (Eds) Historia Física y Política de Chile. Zoología. Maukle and Renou, París 7: 113-320. http://www.biodiversitylibrary.org/item/54589\#3

Blatchley WS (1926) Heteroptera or True Bugs of Eastern North America, with Especial Reference to the Faunas of Indiana and Florida. Nature Publishing Company, Indianapolis. 1116 pp. http://www.biodiversitylibrary.org/item/29937

Champion GC (1897-1901) Insecta: Rhynchota (Hemiptera-Heteroptera), Volume II. In: Godman FD, Salvin O (Eds) Biologia Centrali-Americana, printed by Taylor and Francis, London, xvi + 1-416, 22 plates. [Nabidae: 1899: 297-304; 1900: 305-306]

Cornelis M, Quirán E, Coscarón MC (2012) Description of some immature stages of Nabis (Tropiconabis) capsiformis (Hemiptera: Nabidae). Revista Mexicana de Biodiversidad 83: 1009-1012.

Coscarón MC Nabidae (in press) In: Catalogue of the Heteroptera or true bugs of Argentina. Zootaxa.

Coscarón MC, Volpi LN (2013) Nomenclatural and bibliographic corrections to the Catalog of Nabidae (Hemiptera: Heteroptera) for the Neotropical Region. Zootaxa 3646: 93-96. doi: 10.11646/zootaxa.3646.1.9

Costa A (1853) Cimicum regni Neapolitani centuria. Atti del Reale Istituto d'Incorragiamento alle Scienze Naturali di Napoli 8: 225-299.

Dupuis C (1955) Les génitalia des Hémiptères Héteroptéres. Mémoires du Muséum National d'Histoire Naturelle, Série A Zoologie 6: 183-278.

Germar EF (1837) Hemiptera Heteroptera Promontorii Bonae Spei, Nundum Descripta, Quae Collegit C. F. Drege. Silbermann's Revue Entomologique 5: 121-192.

Google Inc. (2011) Google Earth 6.1.0.4738 (beta) [software program]. http://www.google.es/ intl/es/earth/index.html 
Harris HM (1928) A monographic study of the hemipterous family Nabidae as it occurs in North America. Entomologica Americana 9: 1-97.

Harris HM (1930) Notes on some South American Nabidae. With descriptions of new species (Hemiptera). Annals of the Carnegie Museum 19: 241-248.

Harris HM (1931) Nabidae from the state of Parami. Annals of the Zoological Museum of Polonian 9: 179-185.

Harris HM (1939) Miscelanea sobre Nabidae Sudamericanos. Notas del Museo de La Plata. Tomo IV. Zoologia 26: 368-377.

Henry TJ, Lattin JD (1988) Family Nabidae Costa, 1853. The damsel bugs. In: Henry TJ, Froeschner RC (Eds) Catalog of the Heteroptera, or True Bugs, of Canada and the Continental United States. E. J. Brill, Leiden and New York, 508-520.

Kerzhner IM (1968) New and little know Palaearctic bugs of the family Nabidae (Heteroptera). Entomologicheskoe Obozrenie 47: 848-863 [in Russian]

Kerzhner IM (1987) Neotropical Nabidae (Heteroptera), 2: A new species of Metatropiphorus. Journal New York Entomological Society 95: 596-571.

Kerzhner IM (1993) New and little-known Nabidae from North America. Zoosystematica Rossica 1: 37-45.

Kerzhner IM (2007) Nabidae of the West Indies (Heteroptera). Zoosystematica Rossica 16: $225-234$.

Kerzhner IM, Konstantinov FV (2008) Species of the genus Pagasa from Central and South America (Heteroptera: Nabidae). Zoosystematica Rossica 17: 37-52. http://entomology. bio.pu.ru/personal/konstantinov/Pagasa.pdf

Kiman ZB, Yeargan KV (1985) Development and reproduction of the predator Orius insidiosus (Hemiptera: Anthocoridae) reared on diets of selected plant material and arthropod prey. Annals of the Entomological Society of America 78: 464-67.

Latreille PA (1802) Histoire naturelle, générale et particuliere des Crustacés et des Insectes. Dufart, Paris 3: 1-248.

Lattin JD (1966) Stalia major (Costa) in North America (Hemiptera: Nabidae). Proceedings of the Entomological Society of Washington 68: 314-18.

Lattin JD (1989) Bionomics of the Nabidae. Annual Review of Entomology 34: 383-440. doi: 10.1146/annurev.en.34.010189.002123

Meyer-Dür LR (1870) Entomologishe Parallelen zwischen den Faunen von Central-Europa und der sudamerikanischen Provinz Buenos-Ayres. Mitteilungen der Schweizerischen Entomologischen Gesellschaft 3: 175-178.

Ojeda-Peña D (1971) Biología y hábitos de Nabis capsiformis Germar (Hemip.: Nabidae). Sociedad Entomológica de Perú 14: 297-303.

Pennington MS (1920-1921) Lista de los Hemípteros Heterópteros de la República Argentina. Segunda Parte 17-28. Buenos Aires.

Pericart J (1987) Hémiptères Nabidae d'Europe Occidentale et du Maghreb. Faune France Vol. $71,185 \mathrm{pp}$.

Prado E (2008) Conocimiento actual de Hemiptera-Heteroptera de Chile con lista de especies. Boletín del Museo Nacional de Historia Natural Chile 57: 31-75. 
Reuter OM (1872) Nabidae novae et minus cognitae. Bidrag till Nabidernas Kännedom Af. Öfversigt Kongliga Svenska Vetenskaps-Akademiens Förhandlingar 29: 79-96, plate VIII. Reuter OM (1890) Ad cognitionem Nabidarum. Revue d'Entomologie 9: 289-309. http:// archive.org/details/revuedentomologi910189091soci

Reuter OM (1908) Bemerkungen über Nabiden nebst Beschreibung neuer Arten. Mémoires de la Société Entomologique de Belgique 15: 87-130.

Reuter OM, Poppius B (1909) Monographia Nabidarum orbis terrestris. Acta Societatis Scientiarum Fennicae 37: 1-62.

Ridgway RL, Jones SL (1968) Plant feeding by Geocoris pallens and Nabis americoferus. Annals of the Entomological Society of America 61: 232-233.

Ruffinelli A, Pirán AA (1959) Hemípteros heterópteros del Uruguay. Boletín de la Facultad de Agronomía de Montevideo 51: 1-60.

Signoret V (1863) Revision de Hémiptères du Chile. Annales de la Société Entomologique de France 4: 541-588.

Stål C (1859) Hemiptera: Species novas descripsit. Kongliga Svenska Fregattens Eugenies Resa Omkring Jorden, III (Zoologi, Insekter) 4: 219-298, plates 3-4.

Stål C (1860) Bidrag till Rio de Janeiro Traktens Hemiptera-fauna. Kongliga Svenska Vetenskaps-Akademiens Handlingar 2: 1-84.

Stål C (1862) Bidrag till Rio Janeiro-traktens Hemipterenfauna. II. Kongliga Svenska Vetenskaps-Akademiens Handlingar (N.F.) 3: 1-75.

Stål C (1873) Enumeratio Hemipterorum. Bidrag till en förteckning öfver aller hittills kända Hemiptera, jemte systematiska meddelanden. III. Kongliga Svenska Vetenskaps-Akademiens Handlingar 11: 1-163.

Stoner A (1972) Plant feeding by Nabis, a predaceus genus. Environmental Entomology 1: 557-558.

Volpi LN, Coscarón MC (2010) Catalog of Nabidae (Hemiptera: Heteroptera) for the Neotropical Region. Zootaxa 2513: 50-68. 